Alexander Spencer

\title{
Bild Dir Deine Meinung
}

Die metaphorische Konstruktion des Terrorismus in den Medien

\begin{abstract}
Dieser Artikel möchte eine konstruktivistische Herangehensweise zur Erforschung des Terrorismus illustrieren. Er will zeigen, wie bestimmte metaphorische Konstruktionen des »Terroristen « in den Medien bestimmte Gegenmaßnahmen ermöglichen. Basierend auf Ansätzen aus der kognitiven Linguistik führt der Beitrag eine Metaphernanalyse von Artikeln der Bild-Zeitung durch und arbeitet fünf verschiedene konzeptionelle Verständnisse heraus: 》TERRORISMUS IST KRIEG «; »TERRORISMUS IST VERBRECHEN«; "TERRORISMUS IST UNZIVILISIERT UND BÖSE«; "TERRORISMUS IST EIN NATUREREIGNIS« Und »TERRORISMUS IST KRANKHEIT «. Hierbei zeigt der Artikel, dass Metaphern nicht nur Realität beschreiben, sondern aktiv teilnehmen an der Konstruktion der Welt, wie wir sie sehen, über sie denken und letztendlich auf sie reagieren. Indem Metaphern Verständnisse von einem konzeptuellen Bereich wie Krieg auf andere Bereiche wie Terrorismus übertragen, lassen sie bestimmte Gegenmaßnahmen angemessen erscheinen, während sie andere Möglichkeiten der Entgegnung von vorneherein ausschließen.
\end{abstract}

\section{Einleitung ${ }^{1}$}

Die Disziplin der Internationalen Beziehungen (IB) und die Terrorismusforschung hatten sich in der Vergangenheit nicht besonders viel zu sagen und haben sich relativ unabhängig voneinander entwickelt (Leheny 2002: 58; Spencer 2006: 181). Insbesondere die Terrorismusforschung scheint wenig Interesse an den Theorien der IB zu haben. Wenn man sich die etablierten peer review-Zeitschriften der Terrorismusforschung (Terrorism and Political Violence und Studies in Conflict and Terrorism) ansieht, sind IB-theoretisch orientierte Beiträge Mangelware. Gleichzeitig hat auch die IB offenbar wenig Interesse an der Terrorismusforschung. ${ }^{2}$ Das ist schade, da beide Forschungsfelder einander etwas bieten können. Die IB könnte Einsichten über ein empirisch wichtiges Thema der internationalen Politik erlangen und die Terrorismusforschung könnte von den theoretischen Überlegungen der IB profitieren (Wight 2009: 100).

1 Ich danke Judith Renner, Christopher Daase, Tine Hanrieder, Rainer Hülsse, Alexander Kocks, Christian Kreuder-Sonnen, Andreas Kruck, Jan Tiedemann und Bernhard Zangl für ihre hilfreichen Kommentare und Hilfe bei der Fertigstellung des Artikels. Des Weiteren danke ich der Redaktion der ZIB sowie den anonymen Gutachterinnen und Gutachtern.

2 Die ZIB stellt in dieser Hinsicht keine Ausnahme dar, denn auch hier haben sich nur wenige Beiträge explizit mit Terrorismus oder Terrorismusbekämpfung auseinandergesetzt. Ausnahmen hierfür sind Behr (2004); Behnke (2005 a, b), Herborth (2005), Flügel/Geis (2005) und das Forum zu 9/11 in Zeitschrift für Internationale Beziehungen (2004). 
Insbesondere konstruktivistische Auseinandersetzungen mit Terrorismus sind in der traditionellen Terrorismusforschung sehr selten. ${ }^{3}$ Auch wenn konstruktivistische und/oder diskurstheoretische Untersuchungen von Terrorismus und Anti-TerrorMaßnahmen, insbesondere die Versicherheitlichung von Migration in anderen Bereichen der IB, wie zum Beispiel den Security Studies, existieren (Huysmans 2005: 2; Bigo 2002: 63-66), so werden diese Arbeiten in den Zeitschriften der traditionellen Terrorismusforschung nicht rezipiert. Die Abwesenheit konstruktivistischer Ansätze in der traditionellen Terrorismusforschung ist jedoch überraschend, wenn man in Betracht zieht, dass Worte auch hier schon immer eine wichtige Rolle in der Diskussion über Terrorismus gespielt haben (Onuf 2009: 54). Wenn etwas über die Terrorismusforschung bekannt ist, dann wahrscheinlich, dass sie es bis heute nicht geschafft hat, eine Definition ihres eigenen Forschungsgegenstandes zu entwickeln. All diejenigen, die sich schon einmal mit der Terrorismusthematik beschäftigt haben, wissen um die andauernde Definitionsproblematik (Schmid 1992: 7-11; Daase 2001: 57-59). »Terrorismus« ist ein umstrittener Begriff, ein »essentially contested concept « (Gallie 1956: 169). Darauf mögen einige erwidern: Na und? Wir wissen doch alle ungefähr, was Terrorismus ist, auch wenn wir vielleicht keine einheitliche Wörterbuchdefinition hinbekommen. Ähnlich wie bei Pornographie erkennt man Terrorismus, wenn man ihn sieht (Der Derian 2005: 25; Richardson 2006: 19). So behaupten manche, dass es letztlich egal ist, wie man etwas nennt: Es sind ja nur Worte.

Der vorliegende Beitrag widerspricht diesem Standpunkt. Er argumentiert, dass Sprache und Worte auch in der Terrorismusforschung von Bedeutung sind, da sie der einzige Weg sind, die Welt zu begreifen und ihr Bedeutung beizumessen. Wie in der konstruktivistischen IB schon seit vielen Jahren betont wird, beschreiben Worte nicht nur Realität, sondern nehmen aktiv an der Konstruktion der Welt, wie wir sie sehen, teil und beeinflussen, wie wir auf sie reagieren. Es ist genau diese Art von Realitätskonstruktion und unsere Reaktionen darauf, denen sich dieser Aufsatz widmet. Er leistet zwei zusammenhängende Beiträge: Erstens betont er die Bedeutung von Sprache für die Erforschung von Terrorismus und skizziert, wie eine konstruktivistische Terrorismusforschung aussehen könnte. Dazu greift er, zweitens, auf die Methode der Metaphernanalyse zurück und untersucht, wie gewisse Konstruktionen des »Terroristen« im Diskurs bestimmte Gegenmaßnahmen möglich, logisch und angemessen erscheinen lassen.

Der Aufsatz ist folgendermaßen strukturiert: In Abschnitt zwei erläutert er kurz die Faszination der traditionellen Terrorismusforschung für »harte« Fakten und Feldforschung und verdeutlicht, dass auch die kritische Wende in der Terrorismusforschung ein Faible für Primärquellen behalten hat. Der dritte Abschnitt illustriert eine konstruktivistische, diskursorientierte Variante der Terrorismusforschung jenseits von »harten« Fakten und Feldforschung und zeigt, welche Möglichkeiten die Methode der Metaphernanalyse in der Terrorismusforschung bietet. Der vierte Abschnitt wendet diese konstruktivistische Herangehensweise auf den medialen Diskurs der Bild-

3 Wobei hier die Zeitschrift Critical Studies on Terrorism eine noch sehr junge Ausnahme bildet. 
Zeitung an und zeichnet nach, wie fünf bestimmte metaphorische Konstruktionen (Terrorismus als Krieg, als Verbrechen, als unzivilisiertes Böse, als Naturereignis und als Krankheit) bestimmte Gegenmaßnahmen ermöglichen und andere von vorneherein ausschließen. Der fünfte Abschnitt verweist auf mögliche Anschlussstellen für die weitere Forschung und diskutiert kurz drei mögliche Erklärungsansätze für den Wandel von Metaphern. Das Fazit fasst schließlich das zentrale Argument des Aufsatzes zusammen und reflektiert die Implikationen einer konstruktivistischen Herangehensweise als auch die Möglichkeiten einer Anknüpfung an die traditionelle Terrorismusforschung.

\section{Feldforschung in der traditionellen und kritischen Terrorismusforschung}

Ein Kritikpunkt der traditionellen Terrorismusforschung am eigenen Forschungsfeld ist die unzureichende wissenschaftliche Methodik. So kritisiert Marc Sageman (2008: 14), dass die meisten Terrorismusexperten nie einen Einführungskurs in wissenschaftliches Arbeiten oder ein Seminar zu Forschungsdesigns besucht hätten. Die meisten Bücher seien »oberflächlich und ideologisch« (Wieviorka 1995: 597). Zahlreiche Autoren und Autorinnen behaupten, dieses Problem der unwissenschaftlichen Methodik zeige sich vor allem an der fehlenden Primärquellenforschung (Schmid/ Jongman 1988: 177; Merari 1991: 89-90; Silke 2001: 2). Sie betonen, dass Feldforschung und der direkte Kontakt mit Terroristen essenziell seien für jede wissenschaftliche und objektive Untersuchung von Terrorismus, und dass es keine Alternative zur sorgfältigen Untersuchung von Primärquellen und Feldforschung geben könne (Sageman/Hoffman 2008: 163). Insgesamt, so die selbstkritische Feststellung einiger Vertreterinnen und Vertreter der traditionellen Terrorismusforschung, sei das größte Problem ihres Forschungsfeldes die zu große Abhängigkeit von wiederverwerteten Sekundärquellen. Wie Brendan O'Leary und Andrew Silke (2007: 393) behaupten, haben die meisten »Terrorismusexperten« weder jemals einen Terroristen getroffen noch viel Zeit in einem Konfliktgebiet verbracht. Brian Jenkins (zitiert in Hoffman 2004 a: xviii) vergleicht die Terrorismusforschung diesbezüglich mit Afrikas Kartographen aus dem 19. Jahrhundert, die den Kontinent aus der Ferne abbildeten, ohne jemals dort gewesen zu sein.

Die Gründe für die investigativen Schwierigkeiten und die Abwesenheit von Primärquellenforschung sind klar: Terroristen sind gewalttätig, skrupellos und gefährlich. Die direkte Beobachtung dieser Akteure ist daher voller Risiken für den Forscher und die Forscherin (Toros 2008: 280). Wie Silke (2004: 189) berichtet, wurden bereits einige Forscher und Forscherinnen bei ihren Versuchen Terroristen zu befragen bedroht, entführt, angegriffen und getötet. Zudem wird die Terrorismusforschung, und insbesondere die Primärquellenforschung, erschwert durch die geheime Natur des Forschungsgegenstandes (Silke 2001: 2). Anders als bei anderen Akteuren der internationalen Politik, wie zum Beispiel Politikern der Europäischen Union oder den Vereinten Nationen, ist die Erforschung von Terroristen und deren Organisationen sowie die wissenschaftliche Verifikationsmöglichkeit der Ergebnisse problematisch. 
Autoren wie Ariel Merari (1991: 89-90) argumentieren zudem, dass in-situ-Studien über Terrorgruppen und ihre Strukturen und Prozesse undenkbar seien. Selbst Interviews mit gefangenen oder reumütigen Terroristen sind seiner Meinung nach unbrauchbar, da die Gegebenheiten nicht den »natürlichen Lebensraum« eines Terroristen repräsentierten und diese Art von Stichproben daher nicht repräsentativ sein könne (Merari 1991: 89-90).

Angesichts dieser und anderer Probleme der traditionellen Terrorismusforschung haben mehrere Autoren und Autorinnen in den letzten Jahren eine sogenannte »kritische Wende« in der Terrorismusforschung gefordert (Gunning 2007: 364; Jackson 2007: 244-247) und gründeten dazu auch eine neue Zeitschrift namens Critical Studies on Terrorism. Diese Autoren und Autorinnen, die sich als »kritische Terrorismusforscherinnen und -forscher« begreifen, kritisieren erstens, dass die traditionelle Terrorismusforschung den heutigen Terrorismus als etwas vollkommen Neues klassifiziere und somit Erfahrungen mit Terrorismus in anderen Ländern, Kontexten und Zeitperioden vernachlässige (Breen Smyth 2007: 260). Zweitens wird bemängelt, dass die traditionelle Forschung Einsichten aus anderen Disziplinen, etwa der Anthropologie, Soziologie, Psychologie oder der Friedens- und Konfliktforschung ignoriere (Gunning 2007: 379-380). Drittens wird betont, dass die traditionelle Terrorismusforschung unreflektiert und unkritisch gegenüber dem Staatsterrorismus sei und die Rolle des Staates in der Spirale der Gewalt vernachlässige (Blakely 2007: 228). Viertens wird der traditionellen Terrorismusforschung vorgeworfen, sie habe schwache theoretische Fundamente und ignoriere manche Theorieansätze, wie etwa den Konstruktivismus (Blakeley 2007: 229-231; Jackson 2007: 246). Fünftens wird moniert, dass die traditionelle Terrorismusforschung nur Theorien zur Problemlösung produziere und die Machtstrukturen ignoriere, die Terrorismus überhaupt erst zu einem Problem machten (Jackson 2007: 245). Insgesamt soll eine kritische Wende die Terrorismusforschung sowohl historisch sensibler, interdisziplinärer, skeptischer gegenüber dem Staat als auch theoretischer, konstruktivistischer und reflexiver werden lassen.

Während diese Kritik der kritischen Terrorismusforscher und -forscherinnen durchaus wichtig und berechtigt ist und auch neue Wege zu beschreiten vermag, ist ein sechster Kritikpunkt an der traditionellen Forschung ein altbekannter: Die kritische Terrorismusforschung bemängelt, wie zuvor die traditionelle Forschung auch, dass die meisten Autoren und Autorinnen zu abhängig von Sekundärinformationen seien und nicht genug Primärquellenforschung betreiben würden (Jackson 2007: 244). Beide Forschungsrichtungen, sowohl die kritische als auch die traditionelle, betonen, dass nur der direkte Kontakt mit Terroristen Forschern und Forscherinnen erlaube, Terrorismus besser zu erklären oder zu verstehen. Beide Richtungen plädieren für mehr direkten Kontakt mit »Terroristen«, für Feldforschung vor Ort sowie Interviews und definieren dies als Standard für gute wissenschaftliche Forschung (Breen Smyth 2007: 262; Gunning 2007: 378; Toros 2008: 286). Wie Marie Breen Smyth (2009: 195) betont, ist die nachhaltigste Kritik an der traditionellen Terrorismusforschung der Mangel an Primärquellenforschung und die daraus resultierende derivative Natur vieler Forschungsergebnisse. Primärquellenforschung ist ihrer Meinung nach wich- 
tig, da sie kulturelle und politische Vorurteile und Fehlinterpretationen offenlegen könne. In anderen Worten: »Primary data is a crucial reality-check« (Breen Smyth 2009: 196).

Während die kritische Terrorismusforschung zum einen eine reflexivere, konstruktivistische Herangehensweise bei der Erforschung von Terrorismus fordert, beharrt sie zum anderen darauf, dass nur Primärquellen Einsicht in die »Wahrheit « über Terrorismus geben können (Hülsse/Spencer 2008: 574). Die Forderung nach mehr Primärquellenforschung ist zwar durchaus berechtigt, ich möchte hier jedoch argumentieren, dass Primärquellen nur eine Form von sich gegenseitig beeinflussenden Diskursen über Terrorismus sind. Aus einer konstruktivistischen Perspektive ist die Selbstkonstruktion durch Terroristen genauso Teil dieses Konstruktionsprozesses wie der Diskurs von politischen Eliten oder den Medien.

Deshalb untersucht dieser Artikel Terrorismus aus einer konstruktivistischen Perspektive und widmet sich hier insbesondere den Medien, um zu illustrieren, wie eine konstruktivistische und diskursorientierte Terrorismusforschung aussehen könnte. Der nächste Abschnitt erläutert die Methode der Metaphernanalyse, um diese im darauf folgenden Teil auf den Terrorismusdiskurs in der Bild-Zeitung anzuwenden und zu zeigen, wie dort der »Terrorist« konstruiert wird und welche Gegenmaßnahmen dies ermöglicht.

\section{Eine Methode der Metaphernanalyse}

Ob der Schwerpunkt einer konstruktivistischen Analyse nun auf Akteuren, Strukturen oder deren kodeterminierenden Interaktion basieren soll, ist in der IB kontrovers debattiert worden (Wendt 1987: 337-340; Doty 1997: 367-374; Wight 1999: 113-120; Gould 1998: 82-94). Auch in der Metaphernanalyse gibt es hierzu unterschiedliche Meinungen. Die erste, eher an der Kritischen Diskursanalyse orientierte Auffassung argumentiert, dass Metaphernanalyse versteckte Interessen, Ideologien, Gedanken und Intentionen hinter dem Metapherngebrauch von bestimmten Akteuren aufdecken kann (Fairclough 1992: 194; Musolff 2000: 4). Eine solche kritische Metaphernanalyse will demonstrieren, wie bestimmte Akteure diskursive Strukturen durch den intentionalen Gebrauch von bestimmten Metaphern verändern und wie bestimmte diskursive Praktiken sozio-politische Machtstrukturen reflektieren (Charteris-Black 2004: 29).

Eine zweite, für diesen Artikel zentrale, Auffassung von Metaphernanalyse interessiert sich nicht so sehr für die Akteure und die Intentionen hinter einem bestimmten Metapherngebrauch, sondern mehr dafür, wie die diskursive Struktur Realität fasst und das Handeln von Akteuren ermöglicht beziehungsweise limitiert (Hülsse 2003: 225-226; 2006: 402-405). Diese Herangehensweise steht im Einklang mit vielen anderen diskurstheoretischen wie -analytischen Ansätzen in der IB, wie zum Beispiel denen von David Campbell (1998: 4-7), Roxanne Lynn Doty (1993: 302-305) oder Lene Hansen (2006: 17-36). Unter Rückgriff auf Foucault teilen diese Autorinnen und Autoren ein Diskursverständnis nach dem dieser über den individuellen Dis- 
kursteilnehmern steht. Der Diskurs konstituiert die Akteure und strukturiert, was sie sinnvollerweise sagen und wie sie sinnvollerweise agieren können. Dementsprechend haben Akteure nur eine limitierte agency. Der vorliegende Artikel schließt an dieses Verständnis des Verhältnisses von Akteur und Struktur an und interessiert sich vor allem für die aus Metaphern entstehende diskursive Struktur. ${ }^{4}$ So haben Individuen zwar die kreative Möglichkeit neue Metaphern zu erfinden, doch hängt die Erfolgsaussicht einer solchen Metapher von anderen Sprechern aufgenommen zu werden, stark von ihrer Anknüpfbarkeit an bereits existierende Diskurse und Erfahrungen ab. ${ }^{5}$ Akteure können Metaphern nicht völlig frei intentional nutzen, um den Diskurs zu manipulieren und ihre Interessen zu fördern. Vielmehr sind sie untrennbar in den Diskurs eingebunden und was sie sinnvollerweise sagen können, wird durch die diskursive Struktur bestimmt. Der Einfluss solcher diskursiven Strukturen und das Verhältnis zu Akteuren werden im Folgenden an Hand von Metaphern illustriert.

Metaphernanalysen gehören zu den in den IB am häufigsten genutzten Methoden der Diskursanalyse (Chilton 1996: 47-78; Milliken 1999: 235-236; Spencer 2010: 93-106). Hier werden Diskurse als »utterances« (Neumann 2008: 63) oder als »differential systems of signification« (Milliken 1999: 231) verstanden.

»A discourse, then, is a structure of meaning-in-use that is both intersubjective and, in part, linguistic. It is linguistic in that language is a central sign system that provides the resources out of which representations are constructed. It is intersubjective in that the language through which people construct meaning is necessarily shared «(Weldes/Saco 1996: 373).

Somit kann Diskurs als »virtuelle Textkorpora, deren Zusammensetzung durch im weitesten Sinne inhaltliche (bzw. semantische) Kriterien bestimmt wird « (Busse/ Teubert 1994: 14) gefasst werden. ${ }^{6}$ Das soll nicht heißen, dass Diskurse nur textbasiert sind. Zum Beispiel können Diskurse auch durch Praktiken (Laclau/Mouffe 1985: 113) oder materielle Dinge (Keller/Viehöver 2006: 103) objektiviert werden. Dieser Artikel konzentriert sich jedoch auf die textuelle Seite von Diskursen und insbesondere die in ihnen enthaltene linguistische Figur der Metapher.

Aber was genau versteht man unter einer Metapher? Laut Aristoteles sind Metaphern Übertragungen, bei denen eine Sache mittels einer anderen Sache benannt wird (Mahon 1999: 71). Seitdem ist das Konzept der Metapher in unterschiedlichen Disziplinen immer wieder unterschiedlich detailliert diskutiert worden. Zum Beispiel

4 Was nicht bedeuten soll, dass Agenten der diskursiven Struktur hilflos ausgeliefert wären. Akteure sind auch in der Lage die Struktur, besonders in Momenten der Krise, aktiv zu verändern. Wie in der konstruktivistischen Forschung weitgehend akzeptiert, konstituieren sich Struktur und Akteur gegenseitig, sie bedingen und beeinflussen sich wechselseitig. Die Struktur ermöglicht und limitiert das Handeln von Akteuren, während Akteure die Struktur durch ihr Handeln reproduzieren oder verändern. »[A]ll social action presupposes social structure, and vice versa. An actor can only act socially because there exists a social structure to draw on, and it is only through the action of agents that structure is reproduced « (Dessler 1989: 452). Für eine ausführliche Diskussion der diversen problematiques (Doty 1997: 365) siehe Wendt (1987; 1991; 1992); Dessler (1989); Hollis/Smith (1991; 1992; 1994); Carlsnaes (1992); Jabri/Chan (1996).

5 Bezüglich der Verknüpfung von Metapherngebrauch mit intersubjektiv geteilten Erfahrungen siehe Hülsse/Spencer (2008: 583-585).

6 In diesem Fall ist das inhaltliche Kriterium das Konzept von Terrorist und Terrorismus in der Bild-Zeitung. 
versteht Kenneth Burke (1945: 503) Metaphern als »a device for seeing something in terms of something else« und Susan Sontag (1989: 93) beschreibt Metaphern als »saying a thing is or is like something-it-is not«. So fokussieren sich viele Definitionen auf den etymologischen griechischen Ursprung des Wortes Metapher: Übertragung. Natürlich gibt es eine ganze Reihe von anderen linguistischen Mitteln jenseits von Metaphern, wie zum Beispiel Analogien, Vergleiche oder Metonymien, die eine Bedeutung von einer Sache auf eine andere übertragen. Auch wenn es hier klare Unterschiede gibt, ist es in der Metaphernforschung jedoch weitgehend akzeptiert, den Begriff Metapher als eine Art »all-purpose connector term« (Gozzi 1999: 55) für diese anderen linguistischen Mittel zu benutzen.

In einer rein rhetorischen Perspektive sind Metaphern also vor allem ein »praktisches Label«, um Realität zu beschreiben (Chilton/Lakoff 1999: 56). Sie sind rhetorische Werkzeuge, die nichts weiter tun, als bestimmte Worte mit anderen zu ersetzen, um Sprache zu schmücken. Sie wurden als eine Art »superficial stylistic accessory« verstanden (Beer/Landtsheer 2004: 5). Der vorliegende Artikel möchte jedoch ein anderes, tiefgreifenderes kognitiv-linguistisches Verständnis von Metaphern betonen; ein Verständnis, in dem eine Metapher mehr tut, als einfach ein Wort mit einem anderen zu ersetzen: »Metaphors [...] are devices for simplifying and giving meaning to complex and bewildering sets of observations that evoke concern « (Edelman 1971: 65). Metaphern machen etwas Abstraktes mit Hilfe von etwas Vertrautem verständlich. Dieser kognitiv-linguistische Ansatz, bekannt durch George Lakoff und Mark Johnson (1980), betont die Wichtigkeit von Metaphern für unser Denken und Verstehen der Welt: »[T] he way we think, what we experience and what we do everyday is very much a matter of metaphor « (Lakoff/Johnson 1980: 297). Die Autoren argumentieren, dass Metaphern Menschen dazu bringen, einen konzeptionellen Erfahrungsbereich im Sinne eines anderen zu verstehen, indem sie Wissen aus dem ersten, vertrauten Bereich auf den zweiten, abstrakteren Bereich übertragen. So projizieren Metaphern einen Herkunftsbereich, zum Beispiel KRIEG, auf einen Zielbereich, zum Beispiel TERRORISMUS, und geben so dem Zielbereich einen neuen Bedeutungsgehalt.

Nach Lakoff können zwei Arten von Metaphern unterschieden werden: konzeptionelle Metaphern und metaphorische Ausdrücke. Konzeptionelle Metaphern (zum Beispiel TERRORISMUS IST KRIEG) beinhalten eine abstrakte Verbindung zwischen einem konzeptionellen Bereich und einem Zielbereich (Lakoff 1993: 208-209). Sie bringen uns quasi automatisch dazu, Wissen von einem Herkunftsbereich in einem Zielbereich anzuwenden (Kövecses 2001: 6). Konzeptionelle Metaphern müssen nicht unbedingt direkt im Diskurs sichtbar sein. Sie repräsentieren vielmehr die konzeptuelle Basis oder Idee, die den metaphorischen Ausdrücken zugrunde liegt (Charteris-Black 2004: 9). Metaphorische Ausdrücke hingegen sind die direkt sichtbaren Metaphern, die man im Text findet, etwa: 
»Terror-Krieg gegen Amerika. $\ll^{7}$

"Das ist ein zweites Pearl Harbor. $\ll^{8}$

"Inferno in den USA: Verheerende Terror-Welle mit Tausenden Toten. ${ }^{9}$

Natürlich ist die Formulierung der konzeptionellen Metaphern im Sinne von »A ist $\mathrm{B}$ « ein wenig irreführend, da sie anzudeuten scheint, dass der ganze Zielbereich gleich dem ganzen Herkunftsbereich ist. Dies ist jedoch nicht der Fall, da Konzept A nicht dasselbe ist wie Konzept B. Die Projektion zwischen den beiden Domänen ist immer nur partiell, da nicht alle Charakteristika von Konzept A auf Konzept B übertragen werden. In der Metaphernanalyse ist diese partielle Übertragung weitgehend akzeptiert: »[P]eople make selective distinctions that, by highlighting some aspect of the phenomenon, downplay and hide other features that could give a different stance" (Milliken 1996: 221, Hervorh. dort). Metaphern lenken die Aufmerksamkeit auf einen bestimmten Aspekt eines Phänomens und laden damit Zuhörerinnen und/oder Leser dazu ein, über eine Sache in einem anderen Licht nachzudenken. Insgesamt strukturieren Metaphern, wie Menschen bestimmte Phänomene definieren und beeinflussen so auch ihre Reaktionen auf diese Phänomene (Lakoff 1992: 481; Chilton/Lakoff 1999: 56). Sie limitieren und ermöglichen unsere Handlungsoptionen, da sie die grundlegenden Annahmen und Einstellungen bestimmen, auf denen unsere Entscheidungen basieren (Chilton 1996: 47-78; Milliken 1999: 235-236; Mio 1997: 117-119).

Man muss natürlich vorsichtig sein, wenn man davon spricht, dass Diskurse und insbesondere Metaphern innerhalb eines Diskurses Realität und Politik bestimmen oder verursachen. Wie Richard D. Anderson (2005: 91, Hervorh. dort) betont: $»$ When metaphors are said to cause political phenomena, political science often objects«. Denn letztlich ist es bei einer Metaphernanalyse kaum möglich, »Kausalität« nachzuweisen und daher ist es wichtig zu betonen, dass Metaphern einzelne Politikmaßnahmen nicht in einem positivistisch nachweisbaren Sinne verursachen (Beer/ Landtsheer 2004: 7). Man kann hier nicht von abhängigen und unabhängigen Variablen oder Kausalität, sondern eher von Konstitution sprechen (Wendt 1998: 108-115). Vielmehr eröffnen und verschließen Metaphern Handlungsmöglichkeiten (Shimko 1994: 665). Im Gegensatz zu einer Inhalts- oder Textanalyse stehen die Konsequenzen oder Reaktionen auf die diskursiven Konstruktionen nicht explizit im Text, sondern sind implizit in der Metapher enthalten. Da Metaphern Realität auf eine bestimmte Art konstruieren, definieren sie die Grenzen des gesunden Menschenverstandes, die Grenzen dessen, was als möglich und logisch oder als unmöglich und absurd erachtet wird (Hülsse 2003: 225). Metaphern legen bestimmte Reaktionen nahe und lassen diese somit als ernsthaft denkbar erscheinen.

Wie wird nun aber eine Metaphernanalyse durchgeführt? Hier gibt es eine Reihe von verschiedenen Vorgehensweisen (Gibbs 1999: 29-31; Schmitt 2005: 368-374) und obwohl diese variieren, bleiben die zentralen Schritte ähnlich: Erstens wird ein Textkorpus, wie zum Beispiel Reden von Politikern, Parlamentsdebatten, Fernsehprogramme oder Zeitungsartikel, ausgewählt (Ricœur 1981: 165-171). Zweitens

7 Süddeutsche Zeitung, 12. September 2001: 1, meine Hervorh.

8 Financial Times Deutschland, 12. September 2001: 1, meine Hervorh.

9 Sächsische Zeitung, 12. September 2001: 1, meine Hervorh. 
identifiziert man narrative Elemente, die die Analyseeinheiten, wie zum Beispiel Akteure, Aktionen oder Situationen, für die Metaphernanalyse liefern. Drittens werden metaphorische Ausdrücke, die in dem Textkorpus für die Analyseeinheiten auftauchen, gesammelt. In einem vierten Schritt notiert man häufig wiederkehrende Metaphern und organisiert diese in Clustern, welche dann zu konzeptionellen Metaphern generalisiert werden, die dem Diskurs zugrunde liegen. Je öfter eine Metapher im Diskurs vorkommt, desto mehr Einfluss wird sie auf die Konstitution der Realität und auf die Reaktionen auf diese Realität haben (Charteris-Black 2004: 34). In einem letzten Schritt werden diese Metaphern induktiv interpretiert. Diese Interpretation ist intuitiv und zweifellos subjektiv (Lule 2004: 182). ${ }^{10}$ Es gibt jedoch eine Reihe von Vorschlägen, wie eine Interpretation von Text in einer wissenschaftlichen und kontrollierten Art und Weise durchgeführt werden kann. Eine Möglichkeit bietet hier die von Ronald Hitzler (1993: 230) vorgeschlagene und von Rainer Hülsse (2003: 228) auf Metaphern angewandte Methode der »künstlichen Dummheit«. Durch das Simulieren von Ignoranz über den Zielbereich, zum Beispiel Terrorismus, ist es nun möglich, diesen mit Hilfe des Herkunftsbereichs (z.B. Krieg) zu verstehen. Somit kann man Metaphern interpretieren wie jemand, der diese zum ersten Mal hört (Eco 1995: 191). Mit anderen Worten: Man kann an den Text wie an einen fremdsprachigen herangehen.

Dies gibt daher die Möglichkeit, intuitive Interpretationen von Metaphern anhand von Wörterbüchern zu plausibilisieren, in der Annahme, dass Wörterbücher und Lexika sozial geteiltes Wissen über Phänomene beinhalten. Zum Beispiel findet man für eine Metapher wie »Front« Definitionen in Wörterbüchern, die das Konzept als »die Linie der Kampfberührung von Streitkräften auf dem Kriegsschauplatz« (Meyers Großes Universal Lexikon 1981b: 319) beschreiben, wodurch eine semantische Verbindung zwischen dem Wort »Front« und dem Konzept »Krieg« verdeutlicht wird. Natürlich findet man unterschiedliche Einträge in unterschiedlichen Wörterbüchern, aber die Definitionen sind alle Teile eines bestimmten begrenzten Verständnisbereiches (Pragglejaz Group 2007: 16). Diesbezüglich sind auch user-generierte Enzyklopädien wie Wikipedia hilfreich. Da Beiträge und die sich darin befindlichen semantischen Zusammenhänge und user-generierten Links von allen Nutzern des World Wide Web verfasst oder abgeändert werden können, bieten sie Einsicht in ein sozial weit verbreitetes Verständnis von Konzepten. Der Umstand, dass im Internet viel über die Richtigkeit von Beiträgen gestritten wird, bedeutet, dass die Beiträge tendenziell über Zeit zu einem mehrheitlich akzeptierten Verständnis konvergieren (Pentzold 2007: 45-58; Schlieker/Lehmann 2007: 255-257). Durch diese eher organisch gewachsenen semantischen Beziehungen ist es möglich, eine subjektive Interpretation von Metaphern in eine intersubjektive Interpretation zu verwandeln.

10 Natürlich ist auch schon die Entscheidung, was als metaphorisch erachtet wird und was nicht, eine Interpretation. Hierfür siehe insbesondere Low (1999: 49): »There is always going to be a measure of subjectivity or randomness in identifying expressions.«. 


\section{Die Konstruktion von Terrorismus in der Bild-Zeitung}

Während sich einige Metaphernanalysen hauptsächlich mit den Diskursen politischer Eliten befassen (Ferrari 2007: 615-620; Hülsse 2003: 231-237), interessieren sich andere überwiegend für mediale Diskurse (Pancake 1993: 283-293; Flowerdew/ Leong 2007: 278-290). Dieser Aufsatz wendet sich dem populären Diskurs in den Medien zu und analysiert den Gebrauch von Metaphern in der Bild-Zeitung in Deutschland. Die zentrale Idee dahinter ist, dass solche Medien - und insbesondere die Boulevard-Presse - Einsichten in ein sozial weit verbreitetes Verständnis von Terrorismus geben können. Da nur wenige Menschen Parlamentsdebatten verfolgen oder Politikerreden zuhören, bekommen sie ihre Informationen über die Welt für gewöhnlich aus den Medien. Die Bild ist diesbezüglich besonders wichtig, da sie die größte deutschlandweite Tageszeitung mit über zwölf Millionen Lesern ist. ${ }^{11}$ Es ist daher in der Meinungsforschung unumstritten, dass die Bild zu einem der wichtigsten und mächtigsten Agenda-Setter der Bundesrepublik gehört. ${ }^{12}$ Natürlich werden die Medien durch das beeinflusst, was Eliten sagen, jedoch gilt dies auch umgekehrt und es ist weithin akzeptiert, dass gerade populäre Massenmedien, wie zum Beispiel die Bild, einen sehr großen Einfluss auf die öffentliche Meinung haben. In diesem Artikel dient die Bild-Berichterstattung als Beispiel für einen populären und weit verbreiteten Diskurs über Terrorismus, wobei viele der rekonstruierten Metaphern auch in anderen Zeitungen zu finden sind. ${ }^{13}$ Dies verdeutlichen die folgenden Schlagzeilen aus deutschen Zeitungen am 12.9.2001:

Tabelle 1: Schlagzeilen deutscher Zeitungen nach dem 11. September

\begin{tabular}{|l|l|}
\hline Zeitung & Schlagzeile am 12. September 2001 \\
\hline Süddeutsche Zeitung & Terror-Krieg gegen Amerika \\
\hline Die Tageszeitung & Krieg gegen die USA \\
\hline Rheinische Post & Krieg gegen die Zivilisation \\
\hline Neues Deutschland & Terrorkrieg gegen die USA. Tausende Tote in New York \\
\hline Financial Times Deutschland & »Das ist ein zweites Pearl Harbour« \\
\hline Schweriner Volkszeitung & Die Welt ist schockiert - Krieg gegen die Zivilisation \\
\hline Junge Welt & Kriegserklärung an die USA \\
\hline Express & Krieg gegen Amerika \\
\hline Neue Ruhr Zeitung & Die Welt unter Schock. Krieg gegen die USA \\
\hline Sächsische Zeitung & Inferno in den USA: Verheerende Terror-Welle mit Tausenden Toten \\
\hline Thüringische Landeszeitung & Kriegserklärung gegen die Welt \\
\hline
\end{tabular}

11 Siehe http://www.axelspringer-mediapilot.de/portrait/BILD-BILD_671014.html; 22.03.2011.

12 Siehe http://www.mediatenor.de/charts.php; 25.07.2010.

13 Dies soll nicht bedeuten, dass der untersuchte Diskurs der einzige Diskurs über Terrorismus ist. Für weitere Beispiele siehe Haes (2002: 278-279) und Weller (2005: 322-325). 
Für die Analyse wurde ein Zeitraum von jeweils einem Monat nach insgesamt vier Terroranschlägen in der Zeit zwischen 2001 und 2005 untersucht. Hierfür wurden die vier größten Anschläge ausgesucht: die Anschläge am 11. September 2001 in New York und Washington DC, bei denen etwa 3000 Menschen getötet wurden; die Bombenattentate auf ausländische Touristen in Bali am 12. Oktober 2002, bei denen 202 Menschen ums Leben kamen; die Bombenangriffe auf Pendlerzüge in Madrid am 11. März 2004, bei denen 191 Menschen starben und die Selbstmordangriffe auf den öffentlichen Nahverkehr in London am 7. Juli 2005, bei denen 56 Menschen ihr Leben verloren. Die Studie begrenzte sich aus zwei Gründen auf eine Zeitspanne von einem Monat: Erstens nahm die Anzahl der Artikel über den jeweiligen Anschlag nach einem Monat deutlich ab. Zweitens erbrachte eine Untersuchung über diesen Zeitraum hinaus keine neuen/alternativen konzeptionellen Verständnisse. Nach dem ersten Schritt, der Auswahl des Textkorpus, wurde der Text der Tagesausgabe einer Volllektüre aller Artikel in diesem Zeitraum unterzogen. Hierbei dienten der Terrorist als Akteur, Terrorismus als Aktion und die aus dem Akt resultierende Situation als Analyseeinheiten für die Untersuchung. Beim wiederholten Lesen wurden metaphorische Ausdrücke für diese Analyseeinheiten gesammelt und wiederkehrende Metaphern notiert. In einem nächsten Schritt wurden diese induktiv in thematische Cluster organisiert und zu konzeptionellen Metaphern generalisiert. Wie oben erwähnt, müssen diese konzeptionellen Metaphern nicht explizit im Text auftauchen, sondern entsprechen der thematischen Idee, auf der die metaphorischen Ausdrücke basieren. Die Diskursanalyse identifizierte hier fünf verschiedene konzeptionelle Metaphern: TERRORISMUS IST KRIEG; TERRORISMUS IST VERBRECHEN; TERRORISMUS IST UNZIVILISIERT UND BÖSE; TERRORISMUS IST EIN NATUREREIGNIS und TERRORISMUS IST KRANKHEIT.

\section{Abbildung 1: Zahl der konzeptionellen Metaphern in der Bild ${ }^{14}$}

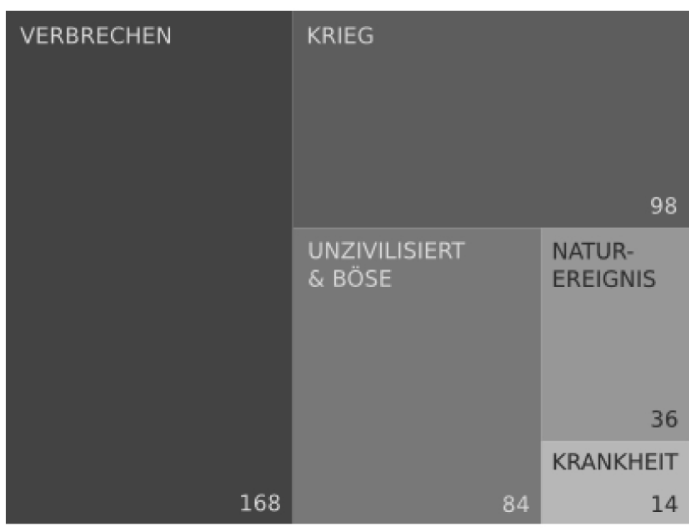

14 Die Anzahl der metaphorischen Ausdrücke ergibt sich aus der Volllektüre von 74 Ausgaben der Bild-Zeitung. 
Die wahrscheinlich bekannteste Metapher versteht Terrorismus als Krieg. Wie aus Abbildungen 1 und 2 ersichtlich, ist die Metapher von Terrorismus als Krieg im Diskurs, besonders nach 9/11, eine weit verbreitete Konstruktion. Außer den »klassischen« Ausdrücken wie »Krieg gegen den Terror« findet man viele metaphorische Ausdrücke, die die Angriffe als Kriegshandlung begreifen. Hier wird zum Beispiel eine Verbindung zum Zweiten Weltkrieg hergestellt, indem 9/11 mit »Pearl Harbor $«{ }^{15}$ verglichen wird oder indem die Attacken als $»$ Kamikaze Angriffe $\ll^{16}$ durch »Kamikaze-Attentäter $«{ }^{17}$ metaphorisiert werden:

»Ein neues Pearl Harbor. Doch diesmal ist nicht die Flotte der USA getroffen worden sondern Amerika, seine Bürger, seine Häuser. Wer redet da noch von Terror? Das ist eine Kriegserklärung aus dem Dunkel. Amerika wird sich wehren, muss sich wehren, wenn es sich nicht aufgeben will. ${ }^{18}$

»Die Kamikaze-Attentäter trafen nicht nur das Herz Amerikas, sondern zerstörten auch den Mythos von CIA und FBI. In den Zwillingstürmen explodierten auch ihre Geheimbüros. >Das ist‘, sagte der republikanische Senator Chuck Hagel geschockt, >ein zweites Pearl Harbor «. «19

Das Verständnis von Terrorismus als Krieg wird bei allen Ereignissen weiter verstärkt durch Metaphern, die die Terroristen als »Al Qaida-Krieger ${ }^{20}$ oder »TerrorKommandos $«{ }^{21}$ in einer $»$ Terroristen-Armee $\aleph^{22}$ konstituieren, in der es nicht nur $»$ Soldaten $\ll^{23}$ sondern auch »kampferfahrene $\ll^{24} »$ Veteranen $\ll^{25}$ gibt.

»Soldaten der Terroristen-Armee marschieren und schwenken ihre Waffen in einem >offiziellen< Video von bin Ladens >al Qaida ${ }^{26}{ }^{26}$

»Afghanistans Schreckensregime hat gestern mit einem Feldzug gegen den Westen gedroht, wenn es angegriffen werde. Das FBI befürchtet, dass Terror-Kommandos des saudischen Milliardärs Osama bin Laden bereits auf dem Weg sind. $^{27}$

15 Bild, 12. September 2001: 1. Die Metapher von »Pearl Harbor« bedeutet für Amerikaner mehr als nur »Krieg «, sondern ist auch verbunden mit Ideen von »Überraschung «, $»$ Verrat « oder »Hinterhältigkeit«. Dies deutet an, wie sehr die Interpretationen von Metaphern vom kulturellen Kontext abhängen. Dazu siehe Littlemore (2003).

Vermerk der Redaktion: Aufgrund der hohen Anzahl an zitierten Artikeln aus der BildZeitung wird auf die Beiträge nur in den Fußnoten und nicht im Literaturverzeichnis verwiesen.

16 Bild, 12. September 2001: 5.

17 Bild, 13. September 2001: 4.

18 Bild, 12. September 2001: 1.

19 Bild, 13. September 2001: 4.

20 Bild, 15. März 2004: 3.

21 Bild, 15. September 2001: 1.

22 Bild, 14. September 2001: 2-3.

23 Bild, 08. Oktober 2001: 3.

24 Bild, 15. September 2001: 5.

25 Bild, 14. September 2001: 2-3.

26 Bild, 08. Oktober 2001: 3.

27 Bild, 15. September 2001: 1. 
»Satelliten-Fotos zeigen sein Versteck inmitten einer steilen, unzugänglichen Bergfestung an der afghanisch-pakistanischen Grenze. Helikopter können dort nicht landen, Motorfahrzeuge nicht fahren. Die Angriffs-Einheiten müssen sich durch 3 Kilometer gefährliches Gebirge kämpfen - gegen eine Terroristen-Armee von ca. 3000 Veteranen aus dem Krieg gegen die russischen Besatzer. Sie sind bewaffnet mit Granaten, Maschinengewehren und Flammenwerfern. $\ll^{28}$

Die Anschläge sind also eine $»$ Kriegserklärung $«^{29}$ an die westliche $» A l l i a n z «^{30}$ und wir befinden uns auf einem »Schlachtfeld « ${ }^{31}$ oder in einem »Kriegsgebiet $« .{ }^{32}$ Wie ein General »kommandiert « ${ }^{33}$ Osama bin Laden mithilfe seiner »Terror-Strategen $\aleph^{34}$ von $»$ Kommando-Zentralen $\iota^{35}$ aus und verteilt $»$ Befehle $\iota^{36}$ an $»$ Terror- $\iota^{37}$ oder $»$ Kampftruppen $\ll{ }^{38}$ Bin Laden hat eine $» P r i v a t$ Armee $«{ }^{39}$ mit einem großen Waffen-»Arsenal «, ${ }^{40}$ welche sich in »Stützpunkten ${ }^{41}$ in Afghanistan versteckt.

»Der Todfeind Amerikas und der freien Welt hat jetzt ganz offiziell dem Westen den Krieg erklärt: Chef-Terrorist Osama bin Laden ließ die von ihm unterzeichnete schriftliche Kriegserklärung durch einen arabischen TV-Sender verbreiten. Er selbst will Truppen der Taliban-Gottes-Krieger in Afghanistan befehligen - angeblich stehen schon 300000 Mann unter Waffen. ${ }^{42}$

»Der Feldzug gegen den Terror hat begonnen. Seit gestern, 18.30 Uhr, wird Afghanistan bombardiert. Kommando-Zentralen von Terror-Chef Osama bin Laden und den TalibanKriegern wurden angegriffen. $\aleph^{43}$

All diese metaphorischen Ausdrücke lassen sich unter dem Konzept Krieg clustern, wenn man sich einige der Definitionen und damit verbundenen Verständnisbereiche in Wörterbüchern ansieht. Die Metapher »Armee« lässt sich etwa verstehen als: »Im engeren Sinn ein für eine bestimmte Aufgabe oder einen Kriegsschauplatz bestimmter Verband unter einheitlichem Oberbefehl « (Meyers Großes Universal Lexikon 1981a: 576). Auch wenn man in Wörterbüchern bei anderen Definitionen wie $»$ Soldat $\aleph^{44}$ oder $»$ Kommando ${ }^{45}$ keinen expliziten Verweis auf Krieg findet, so kön-

28 Bild, 14. September 2001: 2-3.

29 Bild, 25. September 2001: 1.

30 Bild, 25. Oktober 2002: 2.

31 Bild, 14. Oktober 2002: 8.

32 Bild, 12. September 2001: 4.

33 Bild, 17. März 2004: 2.

34 Bild, 06. Oktober 2001: 2.

35 Bild, 08. Oktober 2001: 1.

36 Bild, 13. September 2001: 4.

37 Bild, 28. September 2001: 2.

38 Bild, 25. Oktober 2002: 2.

39 Bild, 19. September 2001: 4.

40 Bild, 19. September 2001: 2.

41 Bild, 21. September 2001: 2.

42 Bild, 25. September 2001: 1.

43 Bild, 08. Oktober 2001: 1.

44 Vgl. Meyers Großes Universal Lexikon (1981d: 132): »militärisch: Angehörige der Streitkräfte eines Staates«.

45 Vgl. Meyers Großes Universal Lexikon (1981c: 50): »ein aus mehreren Soldaten zur Ausführung festgelegter Dienstpflichten oder bestimmter Aufgaben zeitweilig oder ständig gebildetes Organ (z.B. Wach-, Begleitkommando)«. 
nen diese Konzepte dennoch dem semantischen Feld $»$ Krieg « zugeordnet werden. ${ }^{46}$ So lässt sich hier plausibilisieren, dass die oben genannten metaphorischen Ausdrücke der konzeptionellen Metapher TERRORISMUS IST KRIEG zugeordnet werden können.

Wenn man sich nun auf die möglichen Reaktionen im semantischen Feld des Krieges konzentriert, erscheinen eine ganze Anzahl von Möglichkeiten plausibel. Zum Beispiel werden in einem Krieg viele Mittel in die Kriegsanstrengungen gesteckt. Wie Sontag (1989: 99) betont, ist Krieg eine der wenigen Aktivitäten, bei denen man nicht so sehr auf die finanziellen Kosten und den praktischen Nutzen schaut. Krieg ist eine Ausnahmesituation, in der alles für den Sieg mobilisiert wird. Zudem werden Kriege meist mit einer anderen Nation ausgetragen und daher ist es in einem Krieg sinnvoll, seine Grenzen zu sichern und zu befestigen. Menschen aus dem feindlichen Territorium sind automatisch suspekt und werden anders behandelt als die eigene Bevölkerung. Der Ausnahmezustand »Krieg« verlangt nach einer Einschränkung der Gewaltenteilung und macht es unausweichlich, bürgerliche Rechte für den Sieg einzuschränken (Shimko 1994: 658). Insgesamt vereinfacht eine Kriegsmetapher den Sachverhalt, auf den sie projiziert wird. Das Problem wird überschaubar, da es darauf reduziert wird, den Feind zu besiegen und den Krieg zu gewinnen. Die Suche nach den Grundursachen wird diskreditiert und Kritik wird als unpatriotisch, feige und verräterisch verstanden (Hartmann-Mahmud 2002: 429). Die offensichtlichste Forderung dieser Art von Konstruktion ist eine militärische Reaktion (Simon 1987: 9). Wenn ein Land im Krieg steht, muss es militärisch auf einen Angriff reagieren. Eine konzeptionelle Kriegsmetapher lässt eine militärische Reaktion logisch und plausibel erscheinen. Metaphorische Ausdrücke in der Bild wie »Terror-Armee« oder »Soldat« projizieren den Herkunftsbereich »Krieg« auf den Zielbereich »Terrorismus« und konstruieren dadurch den Konflikt mit Al Qaeda als einen Krieg, der durch militärische Mittel, wie zum Beispiel dem Einsatz der Bundeswehr in Afghanistan und die Operation Enduring Freedom, gewonnen werden kann. ${ }^{47}$

\subsection{Terrorismus als Verbrechen}

Eine zweite wichtige konzeptionelle Metapher, die man während des Untersuchungszeitraums in der Bild häufig findet, konstruiert Terrorismus als Verbrechen. Der Terrorist ist also nicht nur ein $»$ Soldat $«$, sondern ein $»$ Verbrecher $«,{ }^{48}$ Al Qaeda ist eine $»$ Bande $«^{49}$ voller $»$ krimineller $«{ }^{50} »$ Mörder $\iota^{51}$ oder $»$ Killer $«:^{52}$

46 Siehe http://de.wikipedia.org/wiki/Soldat oder http://de.wikipedia.org/wiki/Kommando_(Militär); 24.07.2010.

47 Zưr militärischen Anti-Terror Politik Deutschlands siehe Katzenstein (2002); Hyde-Price (2003); Malthaner/Waldmann (2003).

48 Bild, 08. Juli 2005: 3.

49 Bild, 08. Juli 2005: 4.

50 Bild, 17. September 2001: 4.

51 Bild, 12. März 2004: 1.

52 Bild, 14. Juli 2005:8. 
»Mit 33 wurde der Student aus Hamburg zum Mörder von Tausenden Menschen. Wann kam das Böse in seine Seele? «53

»Bei weiteren Durchsuchungen in Leeds - der Heimatstadt der Attentäter - hob die Polizei das Bombenlager der Killer aus, fand eine Badewanne voller Sprengstoff! « ${ }^{54}$

Die »verbrecherischen Anschläge ${ }^{55}$ sind also »mörderische $«^{56} »$ Verbrechen $«, 57$ die nicht nur ein $»$ Kriegsgebiet $«$ sondern auch einen $»$ Mord $\ll^{58}-»$ Tatort $\aleph^{59}$ hinterlassen. ${ }^{60}$ Verantwortlich für diesen $»$ Terrormord $\iota^{61}$ sind nicht nur die $»$ Kommandos $«$, sondern auch die »Täter « ${ }^{62}$ und ihre »Komplizen«. ${ }^{63}$

»Polizisten haben den Tatort abgesperrt. Im zerstörten Gebäude des \Sari-Clubs` waren 350 Urlauber zu Gast. « ${ }^{64}$

»Islamistische Extremisten brachten den Tod in die britische Hauptstadt. Mehr als 50 Tote. Über 700 Verletzte. Die Bomben waren gut versteckt! Niemand konnte dem Inferno entkommen. Einen Tag nach den Terroranschlägen von London ist die Fahndung nach den Tätern und Hintermännern angelaufen. $\ll^{65}$

»Der Kopf der Bahnanschläge von Madrid (191 Tote, 1900 Verletzte) ist tot. Sekunden bevor Spezialkräfte ihn und seine Komplizen festnehmen konnten, sprengten sich die Terroristen in die Luft! « ${ }^{66}$

Wenn man sich nun einige der Definitionen dieser Konzepte ansieht, wird deutlich, dass diese metaphorischen Ausdrücke in einer semantischen Beziehung zu der konzeptionellen Metapher »Verbrechen« stehen: »Mord gilt in allen Rechtsordnungen als gravierendste Straftat gegen das Leben eines Menschen $« .{ }^{67}$ Somit projizieren diese metaphorischen Ausdrücke den Herkunftsbereich VERBRECHEN auf den Zielbereich TERRORISMUS und lassen so den Terroristen in einem besonderen Licht erscheinen.

Im Gegensatz zum Krieg ist Verbrechen etwas Alltägliches. Während ein Krieg Anfang und Ende hat, ist ein Verbrechen ein allgegenwärtiges Phänomen in fast jeder Gesellschaft. Zudem beinhaltet die Konstruktion von Terrorismus als Verbrechen automatisch ein normatives Urteil, welches, ähnlich wie die Metapher »böse«, moralisch aufgeladen ist. Während generell das Recht auf Existenz eines militärischen Gegners anerkannt wird, so ist der Verbrecher immer illegitim (Kappeler/Kappeler

53 Bild, 24. September 2001: 3.

54 Bild, 15. Juli 2005: 7.

55 Bild, 27. März 2004: 2.

56 Bild, 08. Juli 2005: 2.

57 Bild, 25. Oktober 2002: 2.

58 Bild, 23. Oktober 2002: 7 .

59 Bild, 14. Oktober 2002: 9.

60 Die gegenseitige Überlappung von Metaphern und deren Konsequenzen kann hier aus Platzgründen leider nicht weiter thematisiert werden.

61 Bild, 07. April 2004: 1.

62 Bild, 12. September 2001: 1.

63 Bild, 19. Juli 2005: 6.

64 Bild, 14. Oktober 2002: 9.

65 Bild, 09. Juli 2005: 4.

66 Bild, 05. April 2004: 12.

67 http://de.wikipedia.org/wiki/Mord; 23.07.2010, meine Hervorh. 
2004: 176). Während der militärische Gegner uns durchaus ähnlich ist und bestimmte Kriegsregeln befolgt, so ist der Verbrecher hinterlistig. Er bricht Regeln und muss daher bestraft werden. Die Konstruktion von Terrorismus als Verbrechen verlangt also nach polizeilichen oder juristischen Gegenmaßnahmen (Sederberg 1995: 299-300). Mit anderen Worten: Die metaphorische Konstruktion von Terrorismus als etwas Verbrecherisches in der Bild-Zeitung durch Metaphern wie »Mörder « oder »Täter« lässt polizeiliche und legislative Gegenmaßnahmen, wie zum Beispiel die deutschen Sicherheitspakete I und II, angemessen erscheinen. ${ }^{68}$

\subsection{Terrorismus als unzivilisiertes Böse}

Als drittes findet man im Diskurs die konzeptionelle Metapher TERRORISMUS IST UNZIVILISIERTES BÖSE. Hier wird der Terrorist zu einem »barbarischen « ${ }^{69}$ $»$ Bomben-Barbaren $\aleph^{70}$ und der Terrorismus zur $»$ Barbarei $\ll,{ }^{71}$ begangen durch $» u n-$ menschliche $\ll{ }^{72} \gg$ Terror-Bestien $\ll .^{73}$

»Es ist ein Schock für Europa! Nach dem blutigen Bomben-Terror von Madrid führt die Spur - wie nach den Anschlägen von New York, Bali, Djerba und Istanbul - zu Terrorchef Osama bin Laden und seinen barbarischen Al-Qaida-Kriegern! Sie tragen das Blut und die Angst ins Herz Europas. Immer mehr Spuren verweisen auf die islamischen Fanatiker. $\ll^{74}$

Darüber hinaus wird der Terrorist nicht nur als eine $»$ monströse $«{ }^{75} » \mathrm{Hydra}{ }^{76}$ oder ein »Terror-Monster $^{77}$ konstituiert, sondern als Inbegriff des »Bösen «. ${ }^{78}$

»Terror-Monster Osama bin Laden (44) ist auf der Flucht. Der einäugige Taliban-Diktator Omar (Opium-König und Laden-Schwiegersohn) will ihn nicht ausliefern. Bin Laden ist ein Phantom. Viele Infos widersprechen sich. Ist er das kranke Hirn hinter dem schlimmsten Massenmord seit Hitler? « ${ }^{79}$

$68 \mathrm{Zu}$ den juristischen Anti-Terror Maßnahmen in Deutschland siehe Lepsius (2004); Hein (2004); Beckmann (2007); Mauer (2007).

69 Bild, 15. März 2004: 3.

70 Bild, 09. Juli 2005: 4.

71 Bild, 13. September 2001: 2.

72 Bild, 13. September 2001: 6.

73 Bild, 14. September 2001: 1.

74 Bild, 15. März 2004: 3.

75 Bild, 12. September 2001: 6 .

76 Bild, 12. März 2004: 2.

77 Bild, 22. September 2001: 2.

78 Bild, 19. September 2001: 5.

79 Bild, 22. September 2001: 2. 
Der Terrorist ist aber nicht nur ein Phantom, sondern auch der $»$ Teufel $«,{ }^{80} »$ die Verkörperung des Bösen $«,{ }^{81}$ der $»$ teuflische $\aleph^{82} »$ Infernos $«,{ }^{83}$ »apokalyptische $\aleph^{84}$ $»$ Terror-Höllen $«{ }^{85}$ oder gar die $»$ Apokalypse $«{ }^{86}$ selbst verursacht.

»Wer auch immer die Katastrophe von New York oder Washington zu verantworten hat, er hat sich zum Handlanger des Teufels gemacht. ${ }^{87}$

»Es sollte der erste Liebesurlaub mit ihrem Freund werden - für Alexandra K. (27) aus Berlin wurde es eine Reise in die Terror-Hölle. Die Polizei ist nach Auswertung aller Spuren sicher: Alexandra wurde bei dem Anschlag von Bali von der Terrorbombe zerfetzt! $\ll^{88}$

Mithilfe von Wörterbüchern wird bei der Rekonstruktion der Projektion vom Herkunfts- auf den Zielbereich ersichtlich, dass viele der metaphorischen Ausdrücke mit dem Konzept »unzivilisiert « und »böse« semantisch in Verbindung stehen. Zum Beispiel können »Barbaren« als »roh-unzivilisierte, ungebildete Menschen ${ }^{89}$ oder der »Teufel« als die »Personifizierung des Bösen ${ }^{90}$ verstanden werden.

Insgesamt ist die doppelte konzeptionelle Metapher, die den Herkunftsbereich »unzivilisiert « und »böse « auf den Zielbereich »Terrorismus « projiziert, nicht besonders überraschend. Das Phänomen des othering des Feindes war gewalttätigen Konflikten schon immer inhärent (Weller 2001: 4-13; Geis 2006: 148-152). Aber welche »Konsequenzen« haben diese metaphorischen Ausdrücke? Zum Beispiel schließt eine Konstruktion des Terroristen als »böse« die Frage nach den Gründen für ihre Aktionen aus. Warum greifen Terroristen an? Die Antwort ist einfach: Weil sie böse sind. Die Konstruktion von Terrorismus als etwas Böses delegitimiert mit anderen Worten die Forderungen und politischen Ziele der terroristischen Gruppe. Jegliche Gründe für die Gewalt werden ausgeklammert, da »böse« Terroristen nicht für irgendwelche konkreten Motive töten, sondern aus Spaß am Töten. Das Böse wird zur ultimativen Begründung ihrer Taten und gleichzeitig zur Rechtfertigung für extreme Gegenmaßnahmen (Sluka 2009: 145). Zudem wird der Terrorist durch Metaphern wie »Monster« oder »Bestie« entmenschlicht, so dass gewalttätige oder gar tödliche Gegenmaßnahmen gegen Terroristen legitim erscheinen (Ivie 2004: 80). ${ }^{91}$

Die Konstruktion von Terrorismus als »böse« enthält des Weiteren eine klare Polarisierung, da es in diesem Konflikt nur zwei Seiten geben kann: Gut und Böse (Lazar/Lazar 2004: 236). Durch diese Dichotomie wird man selbst automatisch zum Gu-

80 Bild, 21. September 2001: 4.

81 Siehe das Konzept »Teufel«, in: http://www.dwds.de (Das Digitale Wörterbuch der deutschen Sprache des 20. Jahrhundert); 22.07.2010.

82 Bild, 14. Oktober 2002: 8

83 Bild, 08. Juli 2005: 2.

84 Bild, 12. September 2001: 5.

85 Bild, 15. Oktober 2002: 1.

86 Bild, 17. September 2001: 4.

87 Bild, 21. September 2001: 4.

88 Bild, 18. Oktober 2002: 15.

89 http://de.wikipedia.org/wiki/Barbar; 23.07.2010.

$90 \mathrm{http} / / /$ de.wikipedia.org/wiki/Teufel; 22.07.2010.

91 Für das Zusammenspiel von »Bösem« und Terrorismus siehe auch Baudrillard (2003; 2006.). 
ten und die Möglichkeit von Neutralität, die bei einer Metaphorisierung des Terroristen als Soldat noch gegeben wäre, wird ausgeschlossen: Akteure müssen sich für die eine oder andere Seite im Konflikt entscheiden (Ivie 2004: 80). So wird auch das Verhandeln oder jeglicher Dialog mit dem »Bösen« unmöglich, da jeder weiß, dass man keinen Handel mit dem »Teufel« eingehen kann (Abdel-Nour 2004: 429-432).

Dieser Prozess des othering und der Polarisierung ist nicht nur Bestandteil der binären Struktur »Gut versus Böse«, sondern auch der Dichotomie »zivilisiert versus barbarisch«. Auch wenn Barbaren Menschen sein mögen, sind sie trotz allem minderwertig, weniger wissend (»ein völlig ungebildeter Mensch «) $)^{92}$ und weniger kompetent (Kappeler/Kappeler 2004: 182). Sie sind primitiv und gewalttätig (»grausamer Mensch $\ll),{ }^{93}$ man kann nicht rational mit ihnen reden, sie verstehen nur die Sprache der Gewalt (Salter 2002: 163). Vor allem sind Barbaren eines: nicht von hier! Sie sind von außerhalb, wie der griechische Ursprung des Wortes barbaros andeutet, sie sind fremd. So konstruieren Metaphern wie »barbarisch« den Terroristen nicht nur als other, sondern explizit auch als ausländisch, ohne ihm eine Nationalität zuzuweisen (Llorente 2002: 45).

Man kann also schlussfolgern, dass diese Art der Metapher im Diskurs das Verständnis von Fremdheit vom Herkunftsbereich UNZIVILISIERT auf den Zielbereich TERRORISMUS projiziert und dadurch Gegenmaßnahmen, wie zum Beispiel strengere Grenzkontrollen, härtere Einwanderungsbestimmungen oder Antiterrorgesetze logisch erscheinen lassen. Hier ist beispielsweise das deutsche Terrorismusbekämpfungsgesetz von Januar 2002 einschlägig, in dem sich viele Artikel explizit auf Ausländerinnen und Ausländer beziehen (Diez/Squire 2008: 573). Solche Maßnahmen erscheinen angemessen, um die Barbaren vor den Toren zu halten.

\subsection{Terrorismus als Naturereignis}

Die vierte konzeptionelle Metapher, TERRORISMUS IST EIN NATUREREIGNIS, ist etwas ungewöhnlicher als die ersten drei. Hier werden die terroristischen Angriffe als ein $»$ Sturm «, ${ }^{94}$ also als $»$ starker (erheblichen Schaden verursachender) Wind « ${ }^{95}$ oder als eine natürliche $»$ Katastrophe $\iota^{96}$ metaphorisiert:

»Drohen uns neue Selbstmord-Attentate mit Flugzeugen? Aus seinem Versteck in Afghanistan ließ Terroristenchef Osama bin Laden mit einem >Sturm von Flugzeugen / gegen die USA drohen. $\ll^{97}$

92 Siehe das Konzept »Barbar« in: http://www.dwds.de; 22.07.2010.

93 Siehe das Konzept »Barbar« in: http://www.dwds.de; 22.07.2010.

94 Bild, 11. Oktober 2001: 1.

95 Siehe das Konzept $\gg$ Sturm « in: http://www.dwds.de; 22.07.2010.

96 Bild, 15. März 2004: 2.

97 Bild, 11. Oktober 2001: 1. 
»Als glühend die Sonne aufgeht, glitzert Manhattan im Zwielicht der spiegelnden Fenster und der rauchenden Katastrophe. Der Hauch der Katastrophe liegt über der City. Riecht so die Hölle? « ${ }^{98}$

Es wird von einer $»$ Attentats- $«{ }^{99}$ oder $»$ Terrorwelle $«,{ }^{100}$ also einer $»$ sich fortpflanzende[n] auf und ab gehende[n] Bewegung der Wasseroberfläche, besonders des Meeres «, ${ }^{101}$ gesprochen die nicht nur ein $»$ Kriegsgebiet « oder einen $»$ Tatort « hinterlässt, sondern eben auch eine »Katastrophenzone «. ${ }^{102}$

»Erreicht die Terrorwelle jetzt auch Deutschland? Bundesinnenminister Otto Schily sagte gestern: >In Arbeitskreisen von Bund und Ländern werden verschiedene Katastrophenszenarien geprüft ««. ${ }^{103}$

All diese Ausdrücke deuten eine Konstruktion an, die Terrorismus als ein natürliches, unausweichliches Phänomen versteht (Lakoff/Johnson 1980: 145-146; Pancake 1993: 283-284). Wenn wir zum Beispiel Terrorismus als »Welle« metaphorisieren, dann sind Verhandlungen oder Waffenstillstände unsinnig. Eine Welle kann man schließlich kaum davon überzeugen, nicht auf die Küste zu treffen. Als natürliche Ereignisse ist sie unaufhaltbar; sie wird passieren, egal was man versucht. Wenn Terrorismus eine Welle ist, dann werden die Gründe für sein Auftreten unwichtig, da man die physikalischen Gesetze seiner Entstehung ohnehin nicht ändern kann. Klar ist dagegen, dass wir nicht verantwortlich sind für diese Welle. Wie die Definition von »Katastrophe« andeutet, ist es ein »Unglücksfall großen Ausmaßes «. ${ }^{104}$ Während man die Entstehung einer Terrorwelle nicht aufhalten kann, so macht es bei einem solchen Verständnis von Terrorismus dennoch Sinn, Küstenschutzmauern an den Ufern zu verstärken. Oder man bereitet sich auf das unausweichliche Eintreffen der Terrorwelle vor, indem man Institutionen wie das Bundesamt für Bevölkerungsschutz und Katastrophenhilfe (BBK) ${ }^{105}$ kreiert und Katastrophenschutzmaßnahmen implementiert, wie zum Beispiel das Gemeinsame Melde- und Lagezentrum (GMLZ), das sowohl bei Naturkatastrophen wie Überschwemmungen und Stürmen, aber auch bei terroristischen Angriffen die Lage analysiert (Bundesministerium des Inneren 2005: 145-146).

98 Bild, 17. September 2001: 4.

99 Bild, 24. September 2001: 1.

100 Bild, 14. Oktober 2002: 8.

101 Siehe das Konzept »Welle« in: http://www.dwds.de; 22.07.2010.

102 Bild, 08. Juli 2005: 2.

103 Bild, 21. September 2001: 4.

104 Siehe das Konzept $\gg$ Katastrophe« in, http://www.dwds.de; 22.07.2010.

105 Siehe zum Beispiel http://www.bbk.bund.de/nn_402322/DE/01_BBK/BBK_node. html nnn=true; 22.03.2011. 


\subsection{Terrorismus als Krankheit}

Die fünfte konzeptionelle Metapher im Diskurs konstruiert Terrorismus als Krankheit. Hier findet man Metaphern, die Terrorismus als »krank «, ${ }^{106} »$ wahnsinnig « ${ }^{107}$ oder gar als »Pest $«,{ }^{108}$ also eine »epidemisch auftretende Infektionskrankheit, die hohes Fieber und eitrigen Hautausschlag hervorruft und oft tödlich verläuft «, ${ }^{109}$ beschreiben:

»Viele Menschen legen Blumen vor das US-Generalkonsulat. Sonja Hernstorf(28) brachte rote Rosen: >Ich trauere um die vielen toten Menschen, die bei diesem wahnsinnigen Anschlag gestorben sind « ${ }^{110}$

» Der Terror ist die Pest des 21. Jahrhunderts<, sagt Wladimir Putin. Amerika sieht das ebenso und auch die EU. Wie konnte dennoch der Terror die Wahlen in Spanien beeinflussen? Haben die stolzen Spanier sich den Terroristen gebeugt? «111

Indem der Terrorist als ein »Irrer $«^{112}{ }^{12}$ Verrückter $«^{113}$ und somit als $» g e i s t e s-$ krank $«{ }^{114}$ metaphorisiert wird, der in seinem »kranken Kopf « ${ }^{115} »$ Terror-Irrsinn $«{ }^{116}$ plant, wird der Herkunftsbereich KRANKHEIT auf den Zielbereich TERRORISMUS projiziert.

»In seinem kranken Kopf wuchs der Plan vom weltweiten Terror! Jetzt sitzt Doktor Aiman al-Sawahiri (53), Osama bin Ladens Stellvertreter, offenbar in der Falle! Wird er heute verhaftet? «117

Bezüglich der Krankheits-Metaphern argumentiert Ann Mongoven (2006: 413), dass die Assoziation von Terrorismus mit Krankheit dazu beigetragen hat, die hohen zivilen Opfer im Krieg gegen den Terror zu akzeptieren, da sie als notwendige Amputationen gesehen werden, die den Körper vor dem »bösartigen Tumor« des Terrorismus schützen sollen. Des Weiteren ist es plausibel, dass man, ähnlich wie bei den Metaphern »unzivilisiert« und »böse«, mit einer Krankheit, wie zum Beispiel »Wahnsinn«, nicht rational reden und verhandeln kann. Während Abmachungen wie zum Beispiel Waffenstillstandsabkommen oder Friedensverhandlungen mit einem als »Soldaten« verstandenen Terroristen noch möglich erscheinen, sind solche Verhandlungen mit einem »Verrückten« sinnlos. Man kann einem Geisteskranken nicht vertrauen, egal ob es sich um einen »wahnsinnigen« Soldaten oder einen »irren« Verbrecher handelt. ${ }^{118}$ Wie eine psychologische Studie von Emily Pronin et al. (2006: 388-391) gezeigt hat, neigen Menschen weniger dazu, den Gebrauch von Diplomatie

106 Bild, 12. September 2001: 12.

107 Bild, 12. September 2001: 6.

108 Bild, 16. März 2004: 2.

109 Siehe das Konzept »Pest « in, http://www.dwds.de; 22.07.2010.

110 Bild, 12. September 2001: 12.

111 Bild, 16. März 2004: 2.

112 Bild, 26. Oktober 2002: 2.

113 Bild, 12. März 2004: 12.

114 Siehe das Konzept »verrückt« oder »irre« in, http://www.dwds.de; 22.07.2010.

115 Bild, 20. März 2004: 12.

116 Bild, 25. März 2004: 12.

117 Bild, 20. März 2004: 12.

118 Bezüglich der Verbindung von Krankheit und Terrorismus siehe auch Sarasin (2006). 
gegenüber Terroristen zu befürworten, wenn diese als irrational dargestellt werden. Zudem könnte man argumentieren, dass viele Krankheiten wie die »Pest « ansteckend sind und dass sie daher isoliert und in Quarantäne gesteckt werden sollten, weil jeglicher Kontakt mit dem »kranken« Terroristen die Gefahr einer Infektion birgt (Zulaika/Douglass 1996: 62). Insgesamt verschließt also die Konstruktion von Terrorismus als Krankheit die Möglichkeit von direkten Auseinandersetzungen und Verhandlungen mit Terroristen und lässt Versuche, wie zum Beispiel das Versöhnungsangebot von Osama bin Laden im April 2004,119 als unsinnig und gefährlich erscheinen. ${ }^{120}$ So verkündete die Bundesregierung zu dieser Zeit: »Mit Terroristen und Schwerverbrechern wie Osama Bin Laden kann es keine Verhandlungen geben ${ }^{121}$

\section{Trends und Verschiebungen im Metapherngebrauch}

Kernanliegen dieses Artikels war es, die metaphorische Konstruktion von Terrorismus darzustellen und zu zeigen, welche Gegenmaßnahmen ein solches Verständnis logisch und angebracht erscheinen lässt. Der Artikel interessierte sich also für die »Realitäten«, die aus einem bestimmten Metapherngebrauch hervorgehen. Für die zukünftige Forschung drängen sich jedoch zwei weitere Fragen auf: Erstens, woher kommen die Metaphern und warum schaffen es nur bestimmte Konstruktionen, dominant zu werden? Mit anderen Worten: Warum war die konzeptionelle Metapher Terrorismus ist Krieg dominanter als zum Beispiel die in Deutschland fast nicht existente Metapher Terrorismus ist Geschäft (z.B. Al Qaeda als ein Franchise-Unternehmen)? Zweitens, wenn man die Anzahl von Metaphern zwischen 2001 und 2005 genauer betrachtet, wird deutlich, dass es zu einer Verschiebung der Metaphern gekommen ist. So wird in Abbildung 2 ersichtlich, dass der Gebrauch von Kriegsmetaphern nach 9/11 stark abnimmt während der Gebrauch von Verbrechensmetaphern zunimmt. Daher wäre es interessant zu fragen, warum es zu einem solchen Wandel kommt. ${ }^{122}$

Dieser Abschnitt kann nur eine spekulative Antwort auf diese Fragen geben, deren Funktion hier vor allem ist, weitere Forschung zu dem Thema anzuregen. Auch wenn es schwierig oder gar unmöglich ist, spezifische Gründe für den Gebrauch einer be-

119 Siehe http://news.bbc.co.uk/2/hi/middle_east/3628069.stm; 27.07.2010.

120 Agence France Press, »Germany rejects Bin Laden >truce〈 offer«, 15.04.2004; Spiegel Online »Europa steht zusammen«, 15.04.2004; Frankfurter Rundschau, »Bin Laden bietet Frieden; Europäer weisen Angebot ab«, 16.04.2004.

121 Siehe http://archiv.bundesregierung.de/bpaexport/artikel/30/637330/multi.htm; 23.02.2010.

122 Natürlich sind dies nicht die einzigen Fragen, die bezüglich der Rolle von Metaphern bleiben. Eine weitere interessante Frage könnte sich mit der Beziehung zwischen den verschiedenen konzeptionellen Metaphern befassen. Wie stehen die Metaphern zu einander? Sie scheinen sich zu überlappen und teilweise im Widerstreit zu liegen. Zum Beispiel die Metapher »Soldaten« deutet die Möglichkeit von Friedensverhandlungen an, während die Metapher »Wahnsinn« diese auszuschließen scheint. Aus Platzgründen kann hier leider keine tiefgreifende Analyse dieses Zusammenspiels erfolgen. 
Abbildung 2: Verlauf der konzeptionellen Metaphern in der Bild zwischen 2001-2005

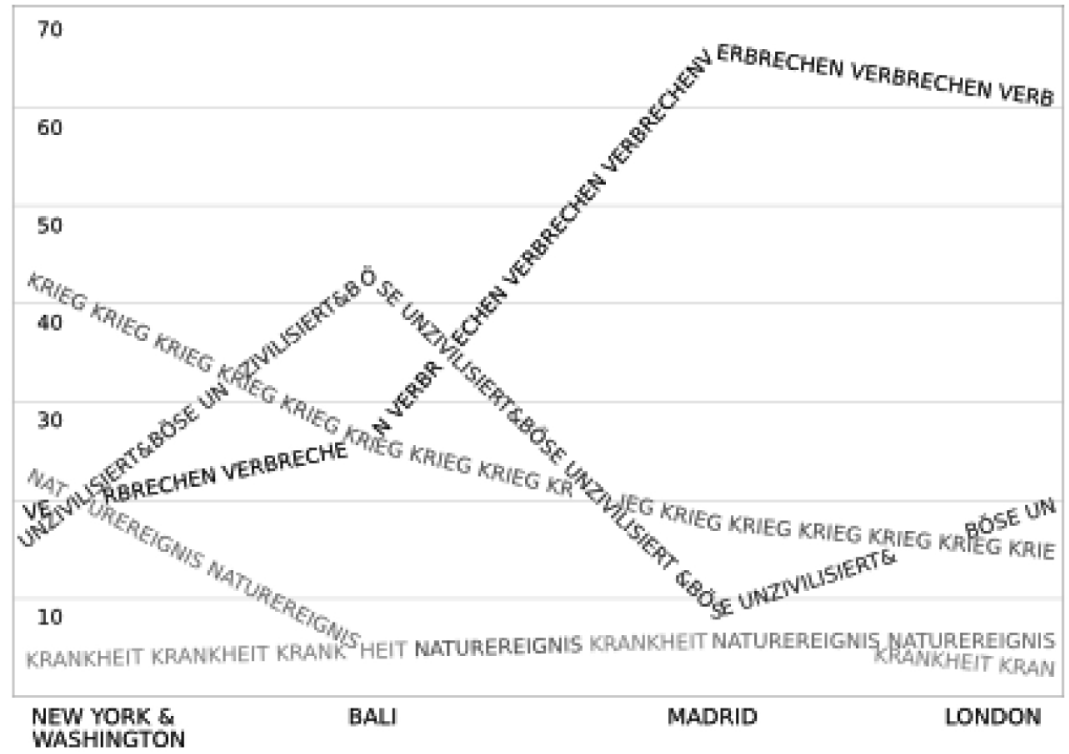

stimmten Metapher herauszuarbeiten, lassen sich drei mögliche Erklärungsrichtungen skizzieren, die jeweils unterschiedliche Annahmen der materialistischen, kritischen und konstruktivistischen Terrorismusforschung widerspiegeln.

Ein materialistischer Erklärungsansatz im Rahmen der traditionellen Terrorismusforschung würde wahrscheinlich auf die »Realität« verweisen und argumentieren, dass eine Verschiebung im Metapherngebrauch die veränderte »Realität« der Gefahrenlage widerspiegelt, die die Metapher beschreiben soll. Da Metaphern hier nur die Funktion zugeschrieben wird, Realitäten zu reflektieren, werden Veränderungen im Diskurs als Resultat von Veränderungen vor Ort verstanden. Zum Beispiel müsste man hier aufzeigen, dass der Terrorismus von Al Qaeda sich "wirklich « von einer militärischen zu einer kriminellen Bedrohung gewandelt hat (Gunaratna 2004: 92-96; Hoffman 2004 b: 551-556).

Eine zweite, kritische Perspektive auf Terrorismus würde eine Verschiebung des Metapherngebrauchs auf die Interessen derer zurückführen, die die Metaphern benutzen. Hier würden also die Veränderungen nicht als Resultat sich ändernder Realitäten gedeutet, sondern als Folge veränderter Interessen der einflussreichen politischen Elite. Man müsste beispielsweise zeigen, wie politische Eliten oder die Medien ihre metaphorische Sprache ändern, um die Öffentlichkeit im Sinne ihrer Interessen zu manipulieren (Drulák/Königova 2007: 18-20). Das beständige Nutzen oder Verändern von Metaphern und damit das implizite Verbreiten von bestimmten Verständnissen, wäre ein expliziter Gebrauch von Macht, der dazu dient, die Macht derjenigen, die solche Metaphern benutzen, zu erhalten oder zu vermehren. Mit anderen Worten: 
Hier werden Metaphern von einem Akteur instrumentell für etwas genutzt. So haben etwa einige Autorinnen und Autoren in Bezug auf den »Krieg gegen den Terror« argumentiert, dass diskursive Mittel und Narrative durch konservative politische Eliten in den Vereinigten Staaten benutzt worden seien, um ihre Interessen zu fördern (Jackson 2005: 2-19). Alternativ könnte eine solche Erklärung für den starken Gebrauch von Kriegsmetaphern nach 9/11 auch auf die finanziellen Interessen der Medien zurückgeführt werden. So könnte man etwa untersuchen, ob der bewusste Gebrauch von Kriegsmetaphern in Medien wie der Bild damit zu erklären ist, dass die Idee des »Krieges « mehr Zeitungen verkauft als andere Metaphern und Verständnisse.

Ein dritter, konstruktivistischer Erklärungsansatz konzentriert sich weniger auf Akteure und interessiert sich mehr für »Erfahrungen«. Hier entstehen oder verändern sich Metaphern, wenn die Logik der Projektion von Herkunfts- auf Zielbereich durch physische und kulturelle Erfahrungen unterstützt wird (Ritchie 2003: 130-133). Wie de Landtsheer und de Vrij (2004: 166) betonen, entstehen Metaphern in einer Krise, weil sie »powerful agents of cognitive framing « sind. Zum Beispiel machten Kriegsmetaphern nach 9/11 in Deutschland Sinn, da dieses Ereignis anscheinend viele Charakteristika mit Erfahrungen von Krieg in der Vergangenheit teilt. Die hohe Opferzahl, das Ausmaß der Zerstörung oder der Gebrauch von Flugzeugen als gesteuerte Bomben machen den Herkunftsbereich Krieg angemessen. Jedoch passten Kriegsmetaphern über Zeit immer weniger zu den Erfahrungen mit Terrorismus in Deutschland. Mit der Abwesenheit weiterer Angriffe ähnlich 9/11 schienen die Erfahrungen von Krieg zunehmend ungeeignet zu sein, um Terrorismus zu konzeptionalisieren und zu verstehen. Metaphern des Verbrechens schienen dagegen angemessener, um die allgemeine Stimmung in Deutschland einzufangen. Anstatt Terrorismus analog zu Krieg als einen außergewöhnlichen Zustand mit hohen Opferzahlen und viel Zerstörung zu erfahren, wurde Terrorismus zunehmend als etwas Normales erachtet. Verbrechensmetaphern waren besser dazu geeignet, die Ansicht zu erfassen, dass Terrorismus etwas Permanentes ist, was uns aber nicht immer konstant und direkt tangiert. Im Unterschied zum ersten Erklärungsansatz, der auf »Realitäten« basiert, werden Metaphern hier nicht als exakte Reflektion von Ereignissen verstanden. Es gibt keine Eins-zu-Eins-Beziehung zwischen Realität und Metaphern, da wir physische Ereignisse nicht direkt beobachten können, sondern dies immer in einem bestimmten interpretativen diskursiven Kontext passiert. Der Diskurs bringt uns dazu, Dinge in einer bestimmten Art und Weise zu sehen, jedoch ist er nicht unabhängig von empirischen Ereignissen. So hatte zum Beispiel die Abwesenheit eines dem 11. September ähnelnden Terrorangriffs in Deutschland Auswirkungen auf den hiesigen Terrorismusdiskurs. Die Logik einer Kriegsmetapher wurde nicht weiter durch physische und kulturelle Erfahrungen unterstützt. Die zentrale Idee dieses Erklärungsansatzes lautet insofern, dass Erfahrungen empirischer Ereignisse Diskurse und gleichzeitig Diskurse Erfahrungen beeinflussen. Anders ausgedrückt: Diskurse und Erfahrungen bedingen sich gegenseitig. 
Fast zehn Jahre nach 9/11 scheint die Terrorismusforschung bereits wieder out zu sein. In Zeiten von Finanzkrise und globalen Umweltproblemen ist Terrorismus nicht mehr das heiße Thema, das er einst war. Dennoch ist die Zukunft der Terrorismusforschung nicht gar so düster, wie sie zunächst erscheinen mag, sofern man alternative Herangehensweisen in Betracht zieht. Dabei gibt es auch klare Anknüpfungspunkte an die traditionelle Terrorismusforschung. Diese hat bereits sehr klar herausgearbeitet, welche Bedeutung die Medien für terroristische Vereinigungen haben und welche Beziehung zwischen beiden existiert (Nacos 1994: 75-93; Wilkinson 2000: 174-187). Hier wurde betont, dass die Medien enorm wichtig waren für Terroristen, da sie so Aufmerksamkeit erlangen und Botschaften an die gesamte Welt richten konnten. Interessanterweise spielen auch hier Metaphern bereits eine wichtige Rolle. Die Medien werden als »Komplizen « (Schmid 1989: 540) oder als die »besten Freunde« (Hoffman 2006: 183) von Terroristen beschrieben und ihre Beziehung wurde als eine »symbiotische beschrieben (Schmid 1989: 540). Wobei die wahrscheinlich bekannteste Metapher Margaret Thatcher zugeschrieben wird: Medien liefern das »oxygen of publicity« (Thatcher zitiert in Wilkinson 2000: 175). Trotz allem gab es bisher sehr wenig wissenschaftliches Interesse an der Wahrnehmung von Terrorismus und an der Frage, welche Rolle Sprache und Diskurse in dieser Darstellung spielen. Dieser Artikel wollte hier einen Beitrag leisten und illustrieren, wie Metaphernanalyse die Terrorismusforschung befruchten kann. Er hat gezeigt, wie bestimmte metaphorische Konstruktionen von Terrorismus bestimmte Gegenmaßnahmen angemessen erscheinen lassen, während sie andere von vornherein ausschließen. Konstruktivistische Ansätze in der Terrorismusforschung sind vielversprechend, da sie alternative Antworten auf traditionelle Fragen offerieren und die Gelegenheit bieten, neue Fragen zu stellen. Wie von den konzeptionellen Metaphern »unzivilisiert « und »böse« oder »Krankheit« angedeutet wird, erscheinen Gegenmaßnahmen, die eine direkte Auseinandersetzung mit dem Terroristen erfordern, absurd oder gar gefährlich. So liegen zum Beispiel Ideen, wie die der Verhandlung oder Versöhnung außerhalb dessen, was als möglich und sinnvoll in Betracht gezogen wird.

\section{Literatur}

Abdel-Nour, Farid 2004: An International Ethics of Evil?, in: International Relations 18: 4, 425-439.

Anderson, Richard D. 2005: The Causal Power of Metaphor: Cueing Democratic Identities in Russia and Beyond, in: Beer Francis A./De Landtsheer, Christ'1 (Hrsg.) Metaphorical World Politics, East Lansing, MI, 91-108.

Baudrillard, Jean 2003: Der Geist des Terrorismus, Wien.

Baudrillard, Jean 2006: Die Intelligenz des Bösen, Wien.

Beckmann, James 2007: Comparative Legal Approches to Homeland Security and Anti-Terrorism, Aldershot. 
Beer, Francis A./De Landtsheer, Christ'l 2004: Metaphors, Politics, and World Politics, in: Beer, Francis A./de Landtsheer, Christ'l (Hrsg.): Metaphorical World Politics, East Lansing, MI, 5-52.

Behnke, Andreas 2005a: 9/11 und die Grenzen des Politischen, in: Zeitschrift für Internationale Beziehungen 12: 1, 117-140.

Behnke, Andreas 2005b: »Ich misstraue allen Systematikern und gehe ihnen aus dem Weg«. Eine Erwiderung auf meine Kritiker, in: Zeitschrift für Internationale Beziehungen 12: 2, 407-415.

Behr, Hartmut 2004: Terrorismusbekämpfung vor dem Hintergrund transnationaler Herausforderungen, in: Zeitschrift für Internationale Beziehungen 11: 1, 27-59.

Bigo, Didier 2002: Security and Immigration: Toward a Critique of the Governmentality of Unease, in: Alternatives 27: 1, 63-92.

Blakeley, Ruth 2007: Bringing the State Back into Terrorism Studies, in: European Political Science 6: 3, 228-235.

Breen Smyth, Marie 2007: A Critical Research Agenda for the Study of Political Terror, in: European Political Science 6: 3, 260-267.

Breen Smyth. Marie 2009: Subjectivities, »Suspect Communities«, Governments and the Ethics of Research on »Terrorism«, in: Jackson, Richard/Breen Smyth, Marie/Gunning, Jeroen (Hrsg.): Critical Terrorism Studies: A New Research Agenda, London, 194-215.

Bundesministerium des Inneren 2005: Zivil- und Katastrophenschutz in Deutschland, in: Bundesministerium des Inneren (Hrsg.): Nach dem 11 September 2001. Maßnahmen gegen den Terror, Berlin, 144-152.

Burke, Kenneth 1945: A Grammar of Motives, Berkley, CA.

Busse, Dietrich/Teubert, Wolfgang 1994: Ist Diskurs ein sprachwissenschaftliches Objekt? Zur Methodenfrage der historischen Semantik, in: Busse, Dietrich/Hermanns, Fritz/Teubert, Wolfgang (Hrsg.): Begriffsgeschichte und Diskursgeschichte. Methodenfragen und Forschungsergebnisse der historischen Semantik, Opladen, 10-28.

Campbell, David 1998: Writing Security: United States Foreign Policy and the Politics of Identity, Minneapolis, MN.

Carlsnaes, Walter 1992: The Agency-Structure Problem in Foreign Policy Analysis, in: International Studies Quarterly 36: 3, 245-270.

Charteris-Black, Jonathan 2004: Corpus Approaches to Critical Metaphor Analysis, Basingstoke.

Chilton, Paul 1996: Security Metaphors: Cold War Discourse from Containment to Common House, New York, NY.

Chilton, Paul/Lakoff, George 1999: Foreign Policy by Metaphor, in: Schäffner, Christina/Wenden, Anita (Hrsg.): Language and Peace, Amsterdam, 37-59.

Daase, Christopher 2001: Terrorismus - Begriffe, Theorien und Gegenstrategien. Ergebnisse und Probleme sozialwissenschaftlicher Forschung, in: Die Friedens-Warte 76: 1, 55-79.

De Landtsheer, Christ'llde Vrij, Ilse 2004: Talking about Srebrenica: Dutch Elites and Dutchbat: How Metaphors Change during Crisis, in: Beer, Francis/de Landtsheer, Christ'l (Hrsg.): Metaphorical World Politics, East Lansing, MI, 163-189.

Der Derian, James 2005: Imaging Terror: Logos, Pathos and Ethos, in: Third World Quarterly 26: 1, 23-37.

Dessler, David 1989: What's at Stake in the Agent-Structure Debate?, in: International Organization 43: 3, 441-473.

Diez, Thomas/Squire, Vicki 2008: Traditions of Citizenship and the Securitisation of Migration in Germany and Britain, in: Citizenship Studies 12: 6, 565-581.

Doty, Roxanne Lynn 1993: Foreign Policy as Social Construction: A Post-Positivist Analysis of U.S. Counterinsurgency Policy in the Philippines, in: International Studies Quarterly 37: 3, 297-320. 
Doty, Roxanne Lynn 1997: Aporia: A Critical Exploration of the Agent-Structure Problematique in International Relations Theory, in: European Journal of International Relations 3: 3, 365-392.

Drulák, Petr/Königove, Lugie 2007: Figuring out Europe: EU Metaphors in the Minds of Czech Civil Servants, in: Perspectives 15: 1, 5-23.

Eco, Umberto 1995: Die Grenzen der Interpretation, München.

Edelman, Murray 1971: Politics as Symbolic Action, Chicago, IL.

Fairclough, Norman 1992: Discourse and Social Change, Cambridge.

Ferrari, Frederica 2007: Metaphor at Work in the Analysis of Political Discourse: Investigating a »Preventive War« Persuasion Strategy, in: Discourse and Society 18: 5, 603-625.

Flowerdew, John/Leong, Solomon 2007: Metaphors in the Discursive Construction of Patriotism: the Case of Hong Kong's Constitutional Reform Debate, in: Discourse and Society 18: 3, 273-294.

Flügel, Oliver/Geis, Anna 2005: Begrenzte Grenzüberschreitung. Eine Replik auf Andreas Behnke, in Zeitschrift für Internationale Beziehungen 12: 2, 391-405.

Gallie, Walter Bryce 1956: Essentially Contested Concepts, in: Proceedings of the Aristotelian Society 56, 167-198.

Geis, Anna 2006: Spotting the »Enemy«? Democracies and the Challenge of the »Other«, in: Geis, Anna/Brock, Lothar/Müller, Harald (Hrsg.): Democratic Wars: Looking at the Dark Side of Democratic Peace, Houndsmills, 142-169.

Gibbs, Raymond 1999: Researching Metaphor, in: Cameron, Lynne/Low, Graham (Hrsg.): Researching and Applying Metaphor, Cambridge, 29-47.

Gould, Harry D. 1998: What Is at Stake in the Agent-Structure Debate?, in: Kubálková, Vendulka/Onuf, Nicholas/Kowert, Paul (Hrsg.): International Relations in a Constructed World, Armonk, NY, 79-98.

Gozzi, Raymond 1999: The Power of Metaphor in the Age of Electronic Media, Creskill, NJ.

Gunaratna, Rohan 2004: The Post-Madrid Face of Al Qaeda, in: The Washington Quarterly 27: 3, 91-100.

Gunning, Jeroen 2007: A Case for Critical Terrorism Studies, in: Government and Opposition 43: 3, 363-393.

Haes, Joachim 2002: Catching the Wave: German Media on September 11, in: Prometheus 20: $3,277-280$.

Hansen, Lene 2006: Security as Practice: Discourse Analysis and the Bosnian War, London.

Hartmann-Mahmud, Lori 2002: War as Metaphor, in: Peace Review 14: 4, 427-432.

Hein, Kirstin 2004: Die Anti-Terrorpolitik der rot-grünen Bundesregierung, in: Harnisch, Sebastian/Katsioulis, Christis/Overhaus, Marco (Hrsg.): Deutsche Sicherheitspolitik. Eine Bilanz der Regierung Schröder, Baden-Baden, 145-171.

Herborth, Benjamin 2005: Zur Politik der Grenzziehung. Eine Replik auf Andreas Behnke, in Zeitschrift für Internationale Beziehungen 12: 2, 383-390.

Hitzler, Ronald 1993: Verstehen: Alltagspraxis und wissenschaftliches Programm, in Jung, Thomas/Müller-Doohm, Stefan (Hrsg.): »Wirklichkeit« im Deutungsprozeß: Verstehen und Methoden in den Kultur- und Sozialwissenschaften, Frankfurt a. M., 223-240.

Hoffman, Bruce 2004a: Foreword, in: Silke, Andrew (Hrsg.): Research on Terrorism: Trends, Achievements and Failures, London, xvii-xix.

Hoffman, Bruce 2004b: The Changing Face of Al Qaeda and the Global War on Terrorism, in: Studies in Conflict and Terrorism 27: 6, 549-560.

Hoffman, Bruce 2006: Inside Terrorism, 2. Auflage, New York, NY.

Hollis, Martin/Smith, Steve 1991: Beware of Gurus: Structure and Action in International Relations, in: Review of International Studies 17: 4, 393-410.

Hollis, Martin/Smith, Steve 1992: Structure and Action: Further Comment, in: Review of International Studies 18: 2, 187-188.

Hollis, Martin/Smith, Steve 1994: Two Stories About Structure and Agency, in: Review of International Studies 20: 3, 241-251. 
Hülsse, Rainer 2003: Sprache ist mehr als Argumentation. Zur wirklichkeitskonstituierenden Rolle von Metaphern, in: Zeitschrift für Internationale Beziehungen 10: 2, 211-246.

Hülsse, Rainer 2006: Imagine the EU: The Metaphorical Construction of a Supra-Nationalist Identity, in: Journal of International Relations and Development 9: 4, 396-421.

Hülsse, Rainer/Spencer, Alexander 2008: The Metaphor of Terror: Terrorism Studies and the Constructivist Turn, in: Security Dialogue 39: 6, 571-592.

Huysmans, Jef 2005: Nexus Terrorism-immigration/Asylum/Refuge in Parliamentary Debates in the UK: Commons debates since 11 September 2001. Report for ESRC Project MIDAS (Migration, Democracy and Security) in the Security Challenges Programme, in: http:// www.midas.bham.ac.uk/Content $\% 20$ analysis $\% 20$ of $\% 20$ Commons $\% 20$ Debates.pdf; 30.06.2009.

Hyde-Price, Adrian 2003: Redefining its Security Role: Germany, in: Buckley, Mary/Fawn, Rick (Hrsg.): Global Responses to Terrorism: 9/11, Afghanistan and Beyond, London, 101-112.

Ivie, Robert L. 2004: Democracy, War, and Decivilizing Metaphors of American Insecurity, in: Beer, Francis A./de Landtsheer, Christ'l (Hrsg.): Metaphorical World Politics, East Lansing, MI, 75-90.

Jabri, Vivienne/Chan, Stephen 1996: The Ontologist Always Rings Twice: Two More Stories About Structure and Agency in Reply to Hollis and Smith, in: Review of International Studies 22: 1, 107-110.

Jackson, Richard 2005: Writing the War on Terror, Manchester.

Jackson, Richard 2007: The Core Commitments of Critical Terrorism Studies, in: European Political Science 6: 3, 244-251.

Kappeler, Victor/Kappeler, Aaron E. 2004: Speaking of Evil and Terrorism: The Political and Ideological Construction of a Moral Panic, in: Deflem, Mathieu (Hrsg.): Terrorism and Counter-Terrorism: Criminological Perspectives, Amsterdam, 175-197.

Katzenstein, Peter J. 2002: Same War, Different Views: Germany, Japan, and the War on Terrorism, in: Current History 101: 659, 427-435.

Keller, Rainer/Viehöver, Willy 2006: Diskursanalyse, in: Behnke, Joachim/Gschwend, Thomas/ Schindler, Delia/Schnapp, Kai-Uwe (Hrsg.): Methoden der Politikwissenschaft, BadenBaden, 103-111.

Kövecses, Zoltan 2002: Metaphor: A Practical Introduction, Oxford.

Laclau, Ernesto/Mouffe, Chantal 1985: Hegemony and Socialist Strategy: Towards a Radical Democratic Politics, London.

Lakoff, George 1992: Metaphor and War: The Metaphor System Used to Justify War in the Gulf, in: Pütz, Marin (Hrsg.): Thirty Years of Linguistic Evolution, Amsterdam, 463-481.

Lakoff, George 1993: The Contemporary Theory of Metaphor, in: Ortony, Andrew (Hrsg.): Metaphor and Thought, 2. Auflage, Cambridge, 202-251.

Lakoff, George/Johnson, Mark 1980: Metaphors We Live By, Chicago, IL.

Lazar, Annita/Lazar, Michelle 2004: The Discourse of the New World Order: »Out-casting « the Double Face of Threat, in: Discourse \& Society 15: 2-3, 223-242.

Leheny, David 2002: Symbols, Strategies, and Choices for international Relations Scholarship After September 11, in: Dialogue IO 1: 1, 57-70.

Lepsius, Oliver 2004: Liberty, Security, and Terrorism: The Legal Position in Germany, in: German Law Journal 5: 5, 435-460.

Littlemore, Jeannette 2003: The Effect of Cultural Background on Metaphor Interpretation, in: Metaphor and Symbol 18: 4, 273-288.

Llorente, Marina A. 2002: Civilization versus Barbarism, in: Collins, John/Glover, Ross (Hrsg.): Collateral Language: A User's Guide to America's New War, New York, NY, 39-51.

Low, Graham 1999: Validating Metaphor Research Projects, in: Cameron Lynne/Low, Graham (Hrsg.): Researching and Applying Metaphor, Cambridge, 48-65.

Lule, Jack 2004: War and its Metaphors: News Language and the Prelude to War in Iraq, 2003, in: Journalism Studies 5: 2, 179-190. 
Mahon, James E. 1999: Getting Your Sources Right. What Aristotle Didn't Say, in: Cameron, Lynne/Low, Graham (Hrsg.): Researching and Applying Metaphor, Cambridge, 69-80.

Malthaner, Stefan/Waldmann, Peter 2003: Terrorism in Germany: Old and New Problems, in: van Leeuwen, Marianne (Hrsg.): Confronting Terrorism: European Experiences, Threat Perceptions and Policies, The Hague, 111-128.

Mauer, Victor 2007: Germany's Counterterrorism Policy, in: Zimmermann, Doron/Wenger, Andreas (Hrsg.): How States Fight Terrorism: Policy Dynamics in the West, London, 59-78.

Merari, Ariel 1991: Academic Research and Government Policy on Terrorism, in: Terrorism and Political Violence 3: 1, 88-102.

Meyers Großes Universal Lexikon 1981a: Armee, Band 1, Mannheim.

Meyers Großes Universal Lexikon 1981b: Front, Band 5, Mannheim.

Meyers Großes Universal Lexikon 1981c: Kommando, Band 8, Mannheim.

Meyers Großes Universal Lexikon 1981d: Soldat, Band 13, Mannheim.

Milliken, Jennifer. L. 1996: Metaphors of Prestige and Reputation in American Foreign Policy and American Realism, in Beer, Francis A./Hariman, Robert (Hrsg.): Post-Realism: The Rhetorical Turn in International Relations, East Lansing, MI, 217-238.

Milliken, Jennifer L. 1999: The Study of Discourse in International Relations: A Critique of Research and Methods, in: European Journal of International Relations 5: 2, 225-254.

Mio, Jeffrey Scott 1997: Metaphor and Politics, in: Metaphor and Symbol 12: 2, 113-133.

Mongoven, Ann 2006: The War on Disease and the War on Terror: A Dangerous Metaphorical Nexus?, in: Cambridge Quarterly of Healthcare Ethics 15: 4, 403-416.

Musolff, Andreas 2000: Mirror Images of Europe: Metaphors in the Public Debate about Europe in Britain and Germany, München.

Nacos, Brigitte 1994: Terrorism and the Media, New York, NY.

Neumann, Iver B. 2008: Discourse Analysis, in: Klotz, Audie/Prakash, Deepa (Hrsg.): Qualitative Methods in International Relations, Basingstoke, 61-77.

O'Leary, Brendan/Silke, Andrew 2007: Conclusion. Understanding and Ending Persistent Conflict: Bridging Research and Policy, in Heiberg, Marianne/O'Leary Brendan/Tirman John (Hrsg.): Terror, Insurgency and the State: Ending Protracted Conflicts, Philadelphia, PA, 387-426.

Onuf, Nicolas 2009: Making Terror/ism, in: International Relations 23: 1, 53-60.

Pancake, Ann S. 1993: Taken by Storm: The Exploitation of Metaphor in the Persian Gulf War, in: Metaphor and Symbol 8: 4, 281-295.

Pentzold, Christian 2007: Wikipedia. Diskussionsraum und Informationsspeicher im neuen Netz, München.

Pragglejaz Group 2007: MIP: A Method for Identifying Metaphorically Used Words in Discourse, in: Metaphor and Symbol 22: 1, 1-39.

Pronin, Emily/Kennedy, Kathleen/Butsch, Sarah 2006: Bombing Versus Negotiating: How Preferences for Combating Terrorism Are Affected by Perceived Terrorist Rationality, in: Basic and Applied Social Psychology 28: 4, 385-392.

Richardson, Louise 2006: What Terrorists Want: Understanding the Enemy, Containing the Threat, London.

Ricour, Paul 1981: Metaphor and the Central Problem of Hermeneutics, in: Thompson, John (Hrsg.): Paul Ric $\propto$ ur: Hermeneutics and the Human Sciences, Cambridge, 165-181.

Ritchie, David 2003: »ARGUMENT IS WAR « - Or Is It a Game of Chess? Multiple Meanings in the Analysis of Implicit Metaphor, in: Metaphor and Symbol 18: 2, 125-146.

Sageman, Marc 2008: Leaderless Jihad, Philadelphia, PA.

Sageman, Marc/Hoffman, Bruce 2008: Does Osama Still Call the Shots?, in: Foreign Affairs 87: 4, 163-166.

Salter, Mark 2002: Barbarians and Civilisation in International Relations, London.

Sarasin, Philipp 2006: Anthrax: Bioterror as Fact and Fantasy, Cambridge. 
Schlieker, Christian/Lehmann, Kai 2007: Verknüpft, verknüpfter, Wikis, in: Lehmann, Kai/ Schetsche, Michael (Hrsg.): Die Google-Gesellschaft. Vom digitalen Wandel des Wissens, Bielefeld, 253-262.

Schmid, Alex 1989: Terrorism and the Media: The Ethics of Publicity, in: Terrorism and Political Violence 1: 4, 539-565.

Schmid, Alex 1992: The Response Problem as a Definitional Problem, in: Terrorism and Political Violence 4: 4, 7-13.

Schmid, Alex/Jongman, Albert 1988: Political Terrorism: A New Guide to Actors, Authors, Concepts, Data Bases, Theories and Literature, Amsterdam.

Schmitt, Rudolf 2005: Systematic Metaphor Analysis as a Method of Qualitative Research, in: The Qualitative Report 10: 2, 358-394.

Sederberg, Peter 1995: Conciliation as Counter-Terrorist Strategy, in: Journal of Peace Research 32: 3, 295-312.

Shimko, Keith L. 1994: Metaphors and Foreign Policy Decision Making, in: Political Psychology 15: 4, 655-671.

Silke, Andrew 2001: The Devil You Know: Continuing Problems with Research on Terrorism, in: Terrorism and Political Violence 13: 4, 1-14.

Silke, Andrew 2004: The Road Less Travelled: Recent Trends in Terrorism Research, in: Silke, Andrew (Hrsg.): Research on Terrorism: Trends, Achievements and Failures, London, 186-213.

Simon, Jeffrey 1987: Misperceiving the Terrorist Threat (RAND Publication Series, R-3423-RC, June 1987), in: http://www.rand.org/pubs/reports/2008/R3423.pdf; 14.11.2008.

Sluka, Jeffrey 2009: The Contribution of Anthropology to Critical Terrorism Studies, in: Jackson, Richard/Breen Smyth, Marie/Gunning, Jeroen (Hrsg.): Critical Terrorism Studies: A New Research Agenda, London, 138-155.

Sontag, Susan 1989: Illness as a Metaphor and AIDS and its Metaphors, New York, NY.

Spencer, Alexander 2006: The Problems of Evaluating Counter-Terrorism, in: UNISCI Discussion Papers 12, 179-201.

Spencer, Alexander 2010: The Tabloid Terrorist: The Predicative Construction of New Terrorism in the Media, Basingstoke.

Toros, Harmonie 2008: Terrorists, Scholars and Ordinary People: Confronting Terrorism Studies with Field Experience, in: Critical Studies on Terrorism 1: 2, 279-292.

Weldes, Jutta/Saco, Diana 1996: Making State Action Possible: The United States and the Discursive Construction of the »The Cuban Problem«, 1960-1994, in: Millennium 25: 2, 361-395.

Weller, Christoph 2001: Feindbilder. Ansätze und Probleme ihrer Erforschung (InIIS-Arbeitspapier 22, Universität Bremen), in: http://www.philso.uni-augsburg.de/lehrstuehle/politik/ politik1/mitarbeiter/weller/pdf_buecher_forschungsberichte/Weller_Feindbilder_01.pdf; 22.03.2011.

Weller, Christoph 2005: Massenmediale Konstruktionen im außenpolitischen Entscheidungsprozess. Die öffentliche Meinung und die deutsche Fernsehberichterstattung am 11. September, in: Ulbert, Cornelia/Weller, Christoph (Hrsg.): Konstruktivistische Analysen der internationalen Politik, Wiesbaden, 313-346.

Wendt, Alexander 1987: The Agent-Structure Problem in International Relations Theory, International Organization 41: 3, 335-370.

Wendt, Alexander 1991: Bridging the Theory/Meta-Theory Gap in International Relations, in: Review of International Studies 17: 4, 383-392.

Wendt, Alexander 1992: Levels of Analysis vs. Agents and Structures: Part III, in: Review of International Studies 18: 2, 181-185.

Wendt, Alexander 1998: On Constitution and Causation in International Relations, in: Review of International Studies 24: 5, 101-117.

Wieviorka, Michael 1995: Terrorism in the Context of Academic Research, in: Crenshaw, Martha (Hrsg.): Terrorism in Context, University Park, PA, 597-606. 
Wight, Colin 1999: They Shoot Dead Horses Don't They? Locating Agency in the Agent-Structure Problematic, in: European Journal of International Relations 5: 1, 109-142.

Wight, Colin 2009: Theorising Terrorism: The State, Structure and History, in: International Relations 23: 1, 99-106.

Wilkinson, Paul 2000: Terrorism versus Democracy: The Liberal State Response, London.

Zeitschrift für Internationale Beziehungen 2004: Forum - Der 11. September und die Folgen für die Disziplin »Internationale Beziehungen«, in: Zeitschrift für Internationale Beziehungen 11: 1, 89-146.

Zulaika, Joseba/Douglass, William A. 1996: Terror and Taboo: The Follies, Fables and Faces of Terrorism, New York, NY. 